\title{
Binary Asteroid Orbit Expansion due to Continued YORP Spin-up of the Primary and Primary Surface Particle Motion
}

\author{
Eugene G. Fahnestock, ${ }^{a, *}$ Daniel J. Scheeres, ${ }^{b}$ \\ aDepartment of Aerospace Engineering, University of Michigan, 2008 FXB Building, 1320 \\ Beal Avenue, Ann Arbor, MI 48109 \\ b Department of Aerospace Engineering Sciences / Colorado Center for Astrodynamics \\ Research, University of Colorado, Engineering Center, ECOT 611, 429 UCB, Boulder, CO \\ 80309 \\ *Corresponding Author E-mail address: efahnest@umich.edu \\ Submitted to Icarus, April 2008
}

\begin{abstract}
:
We examine the hypothesis that within any member of a significant class of close binary asteroid systems with super-synchronously rotating, roughly spheroidal primary and synchronous elongated secondary, continued YORP angular acceleration of the primary causes it to spin at rates where loose material near its equator is lofted from the surface. Subsequent interaction of the material with the secondary causes that material to lose angular momentum and re-impact alpha, but in the process angular momentum is transferred to the orbit causing it to expand. We confirm this hypothesis through precise dynamic and approximate statistical simulation, using the well-characterized 1999 KW4 system model, as KW4 typifies the class of binaries of interest. Our results visibly demonstrate the transfer of angular momentum and our orbit evolution mechanism. In particular, we observe regulation of Alpha spin rate at the rate for which material lofting begins on the same side of Alpha as Beta, but not yet on the opposite side, and nearly constant Alpha angular momentum while the orbit angular momentum grows steadily. The linear fit to that growth is consistent with the YORP torque angular acceleration applied. Lofting occurs in fast transient episodes separated by long periods of slow spin-up under that acceleration. The average amount of material aloft and rate of mass lofting are interesting metrics for the system's lofting activity level contained in our results, but aren't physically descriptive at any particular instant given episodic lofting. We translate the orbit angular momentum growth to average semi-major axis change rate with a simple formula, whose integration also leads to time scales for the system evolution several times faster than standard tidal evolution (such as present orbit size doubling time of $2.46 \mathrm{Myr}$ for KW4). The observationally-supported end state of the system's evolution is likely separation into two asteroids on closely-related heliocentric orbits, with possible shedding of sufficiently more material from the still YORP-torqued, but at that point solitary, primary to form a new secondary and repeat the overall system evolution.
\end{abstract}

Key Words: Asteroids, dynamics; Satellites of Asteroids; Asteroids, surfaces; Regoliths 


\section{Introduction}

YORP has been implicated in the formation of binary asteroids, especially those in a class of close binary asteroids having a large, roughly axisymmetric primary spinning faster than the synchronous rate, and a smaller, roughly triaxial or elongated secondary with on-average synchronous rotation (Pravec et al., 2006). This class includes a large fraction of the binaries found in the NEA population and perhaps in the MBA population as well (Pravec and Harris, 2007).

Once created, the continued evolution of a binary of this type is in question. The usual solid-body tidal evolution should be present, though the parameters are uncertain for rubble-pile bodies like the primary and secondary probably are. Cuk describes another mechanism for such evolution, BYORP, which involves the action of radiation pressure on the secondary (Cuk and Burns, 2005; Cuk, 2007). We describe a different mechanism for binary orbit growth, that of continued YORP spin-up of the primary leading directly to orbit expansion through the transfer of angular momentum due to motion of primary surface particles. Note Cuk et. al. do not include detailed modelling of particle motion or angular momentum transfer between parts of the system in their treatments of their evolution mechanism. Our mechanism fits well with observational results of binary primaries, which are seen to be spinning at or near their "surface disruption limit" (Nolan et al., 2002; Nolan et al., 2004; Shepard et al., 2006; Taylor et al., 2006; Benner et al., 2007). It also predicts orbit expansion times several times faster than those predicted by tidal effects alone. Others are currently investigating this mechanism as well, using quite different models and methodologies (Harris et al., in preparation).

Our hypothesis is simple. Continued YORP angular acceleration of the primary can cause it to spin at rates where loose material on the equator can lift off into orbit. The presence of the secondary, or more properly gravitational interaction between the particles and the secondary, causes these particles to lose angular momentum and re-impact on the surface of the primary, in this way transferring angular momentum to the binary orbit and causing it to grow. On average, we expect all angular momentum deposited by YORP into the primary's spin state that pushes the primary surface beyond the surface disruption limit to eventually be transferred into the orbit. Thus, in a steady-state approximation the orbit angular momentum will grow at a rate equal to the rate that YORP causes the primary's angular momentum to grow. This effect should act as a rotational speed limit on the primary, keeping it rotating at or near its surface disruption limit.

While the hypothesis is simple, there are many assumptions implicit in it that must be verified. This paper performs a series of analyses to flesh out the details of this mechanism and to confirm, with rigorous simulations, that the mechanism works as advertised. For carrying out this confirmation we use the binary system model of asteroid (66391) $1999 \mathrm{KW}$. That system is not only the best characterized asteroid binary and also the best characterized potentially hazardous asteroid (PHA) as large as a kilometer at the date of this writing, but more importantly is a typical member of the class of asynchronous binaries mentioned earlier. General results and conclusions from our confirmation effort focused on KW4 may be extended to all systems in this class.

The first detailed radar observation, shape modelling, and dynamical characterization of a binary near-earth asteroid observed in nature was performed on KW4 (see Ostro et al. (2006) and Scheeres et al. (2006) and the accompanying online supplements). Some very interesting dynamics were revealed for this system, an example of the full two rigid body problem (hereafter F2BP). By way of summary, it was found that KW4 can exist in either a relaxed state or a range of possible excited states. Motions matching four dynamic modes having different periods are more pronounced and more apparent in the excited cases. For reference, the periods are about 2.76 hours (commensurate with the spin period of the larger component, Alpha), about 17.40 hours (commensurate with the mutual orbit period, equal on average to the spin period of the smaller component, Beta), about 188 hours (roughly commensurate with four times the free precession period of Beta), and about 90 days (Scheeres et al., 2006). The motions matching the shorter period modes are mainly librational motion of Beta and variation of in-plane mutual orbit elements, while the motions matching the longest period mode reflect the co-precession of Alpha's spin pole and the mutual orbit plane. A logical next step is to investigate the dynamics of particles, both individually and en masse, within the KW4 system. For this paper, we use the same detailed KW4 component body models that were developed and employed in the prior referenced studies of KW4.

At the core of our evolution mechanism confirmation effort are two types of simulations performed: precise dynamic simulation and approximate statistical (probabilistic) simulation. As for the former; we 
first perform precise dynamic simulation of the F2BP behavior of the binary components by themselves. In section 2 we briefly describe our simulation package to do this propagation, along with some of the underlying equations. Next we perform precise dynamic simulation of batches of non-interacting test particles within the complex, time-varying, full-detail gravity field of the evolving binary. We overview our methodology and underlying equations for this particle propagation in section 3.

Particles naturally originating from the binary components may be ejecta particles leaving the surface at significant velocity relative to the surface, or pieces of material gently lifted up from lying at rest on the surface initially with zero relative velocity. It is the latter type of particle motion (hereafter called "lofting") that is our focus in this paper. It is of interest to determine whether such lofting of particles from the surface of Alpha occurs nearly continuously or episodically, with large or small mass flux per given time period. Lofting is due to Alpha spinning at any rate in excess of the rate at which the total gravitational acceleration acting on particles of regolith lying on the surface (in the equatorial region) equals the centripetal acceleration needed to keep those particles on the surface. Dependent upon how high the spin rate of Alpha is upon release of the material, the motion of lofted particles may result in major regolith redistribution and possibly mass transfer from Alpha to Beta and/or mass loss from the entire system. However, of greater importance are changes to mass, inertia properties, rotation states, and centroid position and velocity for each component of the binary system, and hence changes to the angular momenta of Alpha, Beta, and mutual orbit, in response to each lofted particle's motion.

As the test particles in our precise dynamic simulation, by construction, do not influence each other's motion nor the motion of the binary components, we cannot study the changes to the binary due to the particle's motion using that simulation methodology. Therefore we construct a different probabilistic algorithm for mapping lofted particles forward in time over long durations and simultaneously propagating changes to the states and angular momenta within the binary. We perform post-processing of results from the precise dynamic simulation of particles, both for particle final dispositions (return impact, transfer impact, escape, and lingering in "orbit") and for statistics describing where return impacting particles go in time, position, and relative velocity. This is described in section 4, and the information extracted is used within our probabilistic simulation. That algorithm, also in turn described in section 4, is thus grounded in the outcome of high-fidelity, full-detail dynamics but is freed from the computational constraints of exactly simulating those dynamics.

Our primary results are summarized in section 5. These include observed regulation of Alpha spin rate at the threshold rate where material lofting begins on the same side of Alpha as Beta, but not yet on the opposite side from Beta, and nearly constant Alpha angular momentum and insignificantly minuscule changes to Beta angular momentum while the angular momentum of the orbit grows steadily. The linear fit to that growth is consistent with the YORP torque angular acceleration applied to the primary. Lofting is seen to occur in very fast transient episodes separated by long periods of slow spin-up under the action of the applied acceleration. The amount of material aloft on average and average rate of mass lofting are useful metrics for the activity level of the system, but aren't physically descriptive at any particular instant given the episodic lofting. As overviewed in section 6 , we translate the orbit angular momentum growth to semi-major axis change rate with a simple formula, whose integration also leads to time scales for the system evolution (such as time to double orbit size, over present size, of $2.46 \mathrm{Myr}$ ). We also discuss the observationally-supported end state of the system's evolution, separation into two asteroids on closely-related heliocentric orbits, and discuss possible shedding of sufficiently more material from the still YORP-torqued, but at that point solitary, primary to form a new secondary and repeat the overall system evolution. Finally, we offer some conclusions and next items to address for better fidelity in section 7 .

\section{Binary System Full Two Body Problem Propagation Method}

First we give an overview of the simulation packages employed and their underlying equations, starting with that for the F2BP, a problem characterized by fully coupled rotational and translational (orbit) dynamics. Continuous relative equations of motion (EOM) for the binary system itself are given in Hamiltonian form in Fahnestock and Scheeres (2006). The translational dynamics equations from this can be combined together, and one can perform normalization by a characteristic length scale $L$ (in this case the initial semi-major axis of 2540.5 meters), total system mass $m_{T}=m_{\alpha}+m_{\beta}$ (in this case $2.47 \times 10^{12} \mathrm{~kg}$ ), and a characteristic time scale (we use the mean motion from the above two numbers, i.e. $\sqrt{G\left(m_{\alpha}+m_{\beta}\right) / L^{3}}$ 
). Here and henceforth the subscript ${ }_{\alpha}$ denotes Alpha and ${ }_{\beta}$ denotes Beta, and we generally write vector quantities in bold and dyads and matrices in uppercase roman type. The normalized continuous F2BP EOM, written with respect to the frame fixed to Alpha, become:

$$
\begin{aligned}
& \ddot{\mathbf{r}}+\widetilde{\dot{\boldsymbol{\omega}}_{\alpha}} \mathbf{r}+2 \widetilde{\boldsymbol{\omega}_{\alpha}} \dot{\mathbf{r}}+\widetilde{\boldsymbol{\omega}_{\alpha}} \widetilde{\boldsymbol{\omega}_{\alpha}} \mathbf{r}=\frac{\partial u}{\partial \mathbf{r}} \\
& \mathrm{I}_{\alpha} \dot{\boldsymbol{\omega}}_{\alpha}+\widetilde{\boldsymbol{\omega}_{\alpha}} \mathrm{I}_{\alpha} \boldsymbol{\omega}_{\alpha}=\nu \mathbf{m}_{\alpha} \\
& \mathrm{TI}_{\beta}^{\dot{\omega}} \boldsymbol{\omega}_{\beta}+\widetilde{\boldsymbol{\omega}_{\alpha}} \mathrm{TI}_{\beta} \boldsymbol{\omega}_{\beta}=(1-\nu) \mathbf{m}_{\beta} \\
& \dot{\mathrm{T}}=\mathrm{T} \widetilde{\boldsymbol{\omega}_{\beta}}-\widetilde{\boldsymbol{\omega}_{\alpha}} \mathrm{T} \quad, \quad \dot{\mathrm{P}}=\mathrm{P} \widetilde{\boldsymbol{\omega}_{\alpha}}
\end{aligned}
$$

Here $\mathrm{T}$ is the attitude rotation matrix mapping from the frame of Beta directly to the frame of body Alpha while $\mathrm{P}$ is the rotation matrix mapping from the frame of Alpha to an inertial frame. Note that here $\widetilde{\mathbf{a}}$ represents the skew-symmetric matrix result of the cross product operator acting on vector a, i.e. $\widetilde{(\cdot)}: \mathbb{R}^{3} \mapsto \mathfrak{s o}(3)$ is an isomorphism between $\mathbb{R}^{3}$ and skew-symmetric matrices defined such that $\tilde{\mathbf{x}} \mathbf{y}=\mathbf{x} \times \mathbf{y}$ for any $\mathbf{x}, \mathbf{y} \in \mathbb{R}^{3}$. Here $\mathbf{r}$ is the relative position vector from the centroid of Alpha to the centroid of Beta expressed in the frame of Alpha, the I's are body inertia dyads, and the $\boldsymbol{\omega}$ 's are body angular velocities expressed in their own body frames. We also have the mass parameters of

$$
m=\frac{m_{\alpha} m_{\beta}}{m_{\alpha}+m_{\beta}} \quad, \quad \nu=\frac{m_{\beta}}{m_{\alpha}+m_{\beta}} .
$$

Finally, the moments acting on each body are given by

$$
\begin{aligned}
& \mathbf{m}_{\alpha}=-\widetilde{\mathbf{r}} \frac{\partial u}{\partial \mathbf{r}}-\widetilde{\alpha_{T}} \frac{\partial u}{\partial \alpha_{T}}-\widetilde{\beta_{T}} \frac{\partial u}{\partial \beta_{T}}-\widetilde{\gamma_{T}} \frac{\partial u}{\partial \gamma_{T}} \\
& \mathbf{m}_{\beta}=\widetilde{\alpha_{T}} \frac{\partial u}{\partial \alpha_{T}}+\widetilde{\beta_{T}} \frac{\partial u}{\partial \beta_{T}}+\widetilde{\gamma_{T}} \frac{\partial u}{\partial \gamma_{T}}
\end{aligned}
$$

where $\mathrm{T}=\left[\alpha_{\mathrm{T}}, \beta_{\mathrm{T}}, \gamma_{\mathrm{T}}\right]$. Throughout the above equations, $u$ is the mutual gravitational force potential between the bodies and is dependent on both relative position and relative attitude between them. The formulation of this mutual potential used herein is based on representing each body as a closed polyhedron with triangular faces, and was developed in Werner and Scheeres (2005), with potential derivatives and implementation for simulation presented in Fahnestock and Scheeres (2006).

The above normalized continuous F2BP equations of motion may be integrated using general integration schemes as in Fahnestock and Scheeres (2006). However, for better performance we prefer to use a variational integrator that respects the underlying geometric structure of the configuration space on which the relative dynamics evolve, $\mathrm{SE}(3)=\mathbb{R}^{3}(\mathrm{SO}(3)$. Therefore we use a Lie Group Variational Integrator (LGVI) presented in detail in Lee et al. (2007). This has the advantages of improved total angular momentum and total energy conservation over long simulation durations, improved preservation of the symplectic properties of the system's dynamics, and minimal degradation of the group structure, such as minimal growth in $\left\|\mathrm{T}^{\mathrm{T}} \mathrm{T}-\mathrm{U}\right\|$ where $\mathrm{U}$ is the $3 \times 3$ identity dyad. Additionally, computation time is greatly reduced for the same accuracy when coupled with the polyhedral mutual potential formulation.

We implemented in $\mathrm{C}++$ both single processor and parallel computing versions of both the LGVI's discrete EOM and a general integrator applied to the earlier continuous EOM of Eqs. 1-4. Use of the parallel versions with up to 256 processors in cluster computing environments achieved two-orders-of-magnitude reduction in computation time, in addition to the one-order-of-magnitude reduction from employing the LGVI instead of the higher-order general integration scheme. Our codes have options for including body density variation, including perturbing effects of a third body (as a point mass) on the F2BP dynamics, and changing the number of terms $r$ in the Legendre series expansion at the core of the mutual potential formulation. Without parallelization, the total wall-clock time with our implementation increases as $O\left(n^{2}\right)$ where $n$ is the mean resolution (in number of facets) of the body models, and also increases as $O\left(6^{r}\right)$. Further implementation details are contained in Fahnestock and Scheeres (2008).

Several results sets fully describing the relative motion of Alpha and Beta over time were created with this simulation package. These match different values of the initial osculating orbit elements and orbit-toalpha-spin-pole offset angle that were parametrically varied to achieve different levels of system excitation. 
For the alpha-orbiting particle trajectories examination below, the most excited F2BP results set is used, while for the examination of ejecta motion the most relaxed results set is used, and several F2BP results sets with different parameter values are used for examination of regolith lofting motion.

\section{Restricted Full Three Body Problem Propagation Method}

While the above allows for solution of the detailed motion of the binary system with no influence from any particles, we are interested in the EOM that give the motion of particles themselves, governed by the combined gravity field of the moving binary components. Generally denote $\boldsymbol{\rho}$ as the position vector of a particle. One can write the EOM for it with respect to, and coordinate $\boldsymbol{\rho}$ within, any one of several coordinate frames. In order to match with the usual formulation of the standard restricted three body problem (hereafter R3BP) in which both Alpha and Beta are approximated as point masses in circular orbits about the barycenter, it is helpful to define an "osculating orbit frame" with origin at the system barycenter, i.e. a rotating barycentric frame. At every instant in time this frame has its $+X$ axis fixed to the line of syzygy and pointing from Alpha toward Beta, its $+Z$ axis aligned to the pole of the binary mutual orbit, and $+Y$ axis given by the right hand rule from those axes. Note that when not treating the R3BP system or a similar approximation to the real system, in which the dynamics have no explicit dependence on time, the $+Z$ axis of the rotating barycentric frame and its angular velocity $\boldsymbol{\omega}_{\text {orb }}$ are no longer inertially fixed and constant, respectively.

Assume the same normalizing length scale, mass, and time scale as used above. Then the EOM for a particle in the binary, written with respect to the rotating barycentric frame, are:

$$
\ddot{\boldsymbol{\rho}}+\widetilde{\dot{\boldsymbol{\omega}}_{\mathrm{orb}} \boldsymbol{\rho}}+2 \widetilde{\boldsymbol{\omega}_{\mathrm{orb}}} \dot{\boldsymbol{\rho}}+\widetilde{\boldsymbol{\omega}_{\mathrm{orb}}} \widetilde{\boldsymbol{\omega}_{\mathrm{orb}}} \boldsymbol{\rho}=\frac{\partial u_{\alpha \beta}}{\partial \boldsymbol{\rho}}
$$

The EOM instead written with respect to the Alpha-fixed frame (as may be useful for treating close orbits about Alpha) are:

$$
\ddot{\boldsymbol{\rho}}+\widetilde{\dot{\boldsymbol{\omega}}_{\alpha}} \boldsymbol{\rho}+2 \widetilde{\boldsymbol{\omega}_{\alpha}} \dot{\boldsymbol{\rho}}+\widetilde{\boldsymbol{\omega}_{\alpha}} \widetilde{\boldsymbol{\omega}_{\alpha}} \boldsymbol{\rho}+\ddot{\mathbf{r}}_{\alpha}=\frac{\partial u_{\alpha \beta}}{\partial \boldsymbol{\rho}}
$$

And similarly, for completeness, the EOM written with respect to the Beta-fixed frame are:

$$
\ddot{\boldsymbol{\rho}}+\widetilde{\dot{\boldsymbol{\omega}}_{\beta}} \boldsymbol{\rho}+2 \widetilde{\boldsymbol{\omega}_{\beta}} \dot{\boldsymbol{\rho}}+\widetilde{\boldsymbol{\omega}_{\beta}} \widetilde{\boldsymbol{\omega}_{\beta}} \boldsymbol{\rho}+\ddot{\mathbf{r}}_{\beta}=\frac{\partial u_{\alpha \beta}}{\partial \boldsymbol{\rho}}
$$

Here the $\ddot{\mathbf{r}}_{\alpha}$ denotes the inertial acceleration of the centroid Alpha and similarly for Beta. Finally, and most simply, there are the EOM in the inertial barycentric frame:

$$
\ddot{\boldsymbol{\rho}}=\frac{\partial u_{\alpha \beta}}{\partial \boldsymbol{\rho}}
$$

In each case, the position vector $\boldsymbol{\rho}$ is coordinated in the appropriate frame of the EOM.

Throughout the above equations, $u_{\alpha \beta}$ is the total force potential at a field point due to the mass distributions of both Alpha and Beta. Depending on the model of the system used, $u_{\alpha \beta}$ may become simpler or more complicated, as it does with the choice of different frames and hence different EOM. We state here the form of $u_{\alpha \beta}$ only for those cases of greatest relevance. For the R3BP (in which both Alpha and Beta are approximated as perfect spheres), this force potential for the rotating barycentric frame EOM is

$$
u_{\alpha \beta}=(1-\nu) \frac{1}{\sqrt{(x+\nu)^{2}+y^{2}+z^{2}}}+\nu \frac{1}{\sqrt{(x-(1-\nu))^{2}+y^{2}+z^{2}}} .
$$

If Alpha is approximated as a sphere but Beta is approximated as a triaxial ellipsoid with long axis locked to the line of syzygy at all times, so that Beta's rotation is synchronous with the mutual orbit, then we have what we call the RSE3BP (restricted sphere+ellipsoid three body problem). In this case, the force potential for the rotating barycentric frame EOM is

$$
u_{\alpha \beta}=(1-\nu) \frac{1}{\sqrt{(x+\nu)^{2}+y^{2}+z^{2}}}+\nu u_{\text {ellip }}(x-(1-\nu), y, z)
$$


where $u_{\text {ellip }}$ is the standard ellipsoid potential result, calculated in the fashion indicated in equations 11-13 of Bellerose and Scheeres (2007), but with the normalization length scale of $L$ rather than the largest semi-axis, and hence the normalized largest semi-axis no longer equal to one. These expressions are not repeated here for brevity. Finally, if instead we have each binary component represented as a full polyhedral mesh with almost arbitrary shape, then we have the RF3BP (restricted, since the particle mass is still ignored and can be considered $\approx 0$, but "full", in detail of the shape and gravity fields of the binary components, three body problem). For any set of EOM, the force potential is then

$$
\begin{aligned}
u_{\alpha \beta}= & -G \rho_{\alpha}\left(-\frac{1}{2} \sum_{\text {efedges in Alpha }} \mathbf{r}_{e_{\alpha}} \cdot \mathrm{E}_{e_{\alpha}} \cdot \mathbf{r}_{e_{\alpha}} L_{e_{\alpha}}+\frac{1}{2} \sum_{f \in \text { faces in Alpha }} \mathbf{r}_{f_{\alpha}} \cdot \mathrm{F}_{f_{\alpha}} \cdot \mathbf{r}_{f_{\alpha}} \omega_{f_{\alpha}}\right) \\
& -G \rho_{\beta}\left(-\frac{1}{2} \sum_{\text {efedges in Beta }} \mathbf{r}_{e_{\beta}} \cdot \mathrm{E}_{e_{\beta}} \cdot \mathbf{r}_{e_{\beta}} L_{e_{\beta}}+\frac{1}{2} \sum_{f \in \text { faces in Beta }} \mathbf{r}_{f_{\beta}} \cdot \mathrm{F}_{f_{\beta}} \cdot \mathbf{r}_{f_{\beta}} \omega_{f_{\beta}}\right)
\end{aligned}
$$

Here $G$ is the normalized gravity constant (for our problem, unity), and likewise the assumed constant scalar body densities $\rho_{\alpha}$ and $\rho_{\beta}$ are also normalized. All of the vector, dyad, and scalar quantities within the summations over every face and every edge of the polyhedron representing each body are as defined in Werner and Scheeres (1997). Expressions for these quantities are also not repeated here for brevity. It is important to note that the relative position vector of the particle in the frame fixed to the respective body is required within the quantities $\mathbf{r}_{e}, \mathbf{r}_{f}, L_{e}$ and $\omega_{f}$. Hence we use the transpose of attitude rotation matrices $\mathrm{R}_{\alpha}$ and $\mathrm{R}_{\beta}$, which map from the frame of Alpha to the frame of the EOM and from the frame of Beta to the frame of the EOM respectively, to convert the position vector $\boldsymbol{\rho}$ within the EOM into each body frame. These rotation matrices then show up in the partial derivatives of the potential with respect to $\boldsymbol{\rho}$. For the force on the particle, we have the first partial:

$$
\begin{aligned}
& \frac{\partial u_{\alpha \beta}}{\partial \boldsymbol{\rho}}=-G \rho_{\alpha} \mathrm{R}_{\alpha}\left(\sum_{\text {efedges in Alpha }} \mathrm{E}_{e_{\alpha}} \cdot \mathbf{r}_{e_{\alpha}} L_{e_{\alpha}}-\sum_{f \in \text { faces in Alpha }} \mathrm{F}_{f_{\alpha}} \cdot \mathbf{r}_{f_{\alpha}} \omega_{f_{\alpha}}\right) \\
& -G \rho_{\beta} \mathrm{R}_{\beta}\left(\sum_{\text {efedges in Beta }} \mathrm{E}_{e_{\beta}} \cdot \mathbf{r}_{e_{\beta}} L_{e_{\beta}}-\sum_{f \in \text { faces in Beta }} \mathrm{F}_{f_{\beta}} \cdot \mathbf{r}_{f_{\beta}} \omega_{f_{\beta}}\right)
\end{aligned}
$$

And for the gravity gradient matrix, or the second partial, we have:

$$
\begin{aligned}
& \frac{\partial^{2} u_{\alpha \beta}}{\partial \boldsymbol{\rho}^{2}}=-G \rho_{\alpha} \mathrm{R}_{\alpha}\left(\sum_{\text {efedges in Alpha }} \mathrm{E}_{e_{\alpha}} L_{e_{\alpha}}-\sum_{f \in \text { faces in Alpha }} \mathrm{F}_{f_{\alpha}} \omega_{f_{\alpha}}\right) \mathrm{R}_{\alpha}^{T} \\
& -G \rho_{\beta} \mathrm{R}_{\beta}\left(\sum_{e \in \text { edges in Beta }} \mathrm{E}_{e_{\beta}} L_{e_{\beta}}-\sum_{\text {fefaces in Beta }} \mathrm{F}_{f_{\beta}} \omega_{f_{\beta}}\right) \mathrm{R}_{\beta}^{T}
\end{aligned}
$$

Also, it should be noted that the Laplacian of the potential from each body individually is given by

$$
-G \rho_{i} \sum_{f \in \text { faces in body } i} \omega_{f_{i}}, \quad i \in(\alpha, \beta)
$$

Our simulation package for particle propagation makes use of the RF3BP formulation, while the R3BP and RSE3BP formulations limited to just the rotating barycentric frame EOM are useful for some analytical purposes (see below). It was originally intended for our single-processor $\mathrm{C}++$ implementation of the RF3BP formulation to be flexible enough to propagate any of the four sets of particle EOM for the four frames. However, only the barycentric inertial frame EOM are propagated for the results herein. It was also intended to have options in the code for driving the motions of Alpha and Beta in several ways: 1) by interpolating the raw state data files output from prior full-detail F2BP simulation of KW4; 2) by moving the components according to periodic functions tuned to the frequencies of the four dynamic modes, as measured from 
looking at prior full-detail F2BP simulation of KW4; 3) by propagating concurrently with the particle EOM other equations of motion for the binary itself using approximate (but fast to compute) 2nd-degree-and-order gravity representations of each component. Only the first of these methods (having the highest fidelity) is used for the particle motion results herein. Finally, it should be noted that impact detection is implemented using the Laplacian for each body (which switches from zero at particle positions outside a body polyhedron to $-4 \pi$ at particle positions interior to that polyhedron).

\section{Modelling of Primary Regolith Particle Lofting}

\subsection{Full dynamic simulation of regolith particles}

Several F2BP motion results sets are used as underlying binary motion for dynamic simulation of lofted particles through the system. These results sets match with $0.0^{\circ}, 2.5^{\circ}$, and $5.0^{\circ}$ of initial pole offset angle and with $0^{\circ}$ and $180^{\circ}$ of initial phase of the chosen lofting facet w.r.t. the syzygy plane (the plane formed by the Alpha spin pole and line of syzygy), measured counterclockwise from the Beta side of Alpha. While all of these results sets are generated using an initial Alpha spin rate of $0.000651444 \mathrm{rad} / \mathrm{s}$, the set for $2.5^{\circ}$ pole offset and $180^{\circ}$ phase (for opposite side lofting) is also generated a second time with the initial Alpha spin rate of $0.000641444 \mathrm{rad} / \mathrm{s}$, while that for $2.5^{\circ}$ offset but $0^{\circ}$ phase (for same side lofting) is also regenerated with the initial Alpha spin rate of $0.000640444 \mathrm{rad} / \mathrm{s}$.

An explanation is in order for this choice of parameters and for why the initial Alpha angular velocity, aligned to the Alpha $+Z$ axis, is given these magnitudes, rather than the best estimated observation-derived spin rate for KW4 Alpha (0.000631343 rad/s Ostro et al. (2006)). The parameters come from an effort to identify the exact location on Alpha's surface and exact binary system conditions at which lofting would most likely first occur under YORP-driven primary spin-up. Here 'binary system conditions' include Alpha spin rate, mutual orbit elements, pole offset angle, Beta libration angle, and relative pose in phase angle of the above location on Alpha's surface from the syzygy plane, and so on.

First looking at the uniformly spinning Alpha model by itself, we calculate for every face the angular velocity at which lofting will first occur (as angular velocity is slowly increased) at any point on that face , and the angular velocity at which lofting should first be guaranteed to occur at every point on that face. This is plotted in Figure 1, for most all facets not lying at extreme latitudes (i.e. near the north pole, and thus having very low face ID \#'s, or near the south pole, and thus having very high face ID \#'s approaching the total Alpha face count of 9168). As seen in the enlarged panel, near equatorial face \# 4113 had the lowest guaranteed-everywhere-lofting spin rate, equal to $0.000651444 \mathrm{rad} / \mathrm{s}$ (about $3.18 \%$ higher than the observation-derived spin rate). Simultaneously, the same face \# 4113's first-lofting spin rate is almost exactly the observation-derived spin rate. Thus we adopt this face as our chosen lofting facet, and determine that suitable threshold rates at which lofting might be considered to "turn on" there, for purposes of the model of the next section, should be chosen about halfway between the first-lofting and guaranteed-everywhere-lofting spin rates for this face.

Next we move from the model of uniformly spinning Alpha by itself to a toy model for the full binary system, having both full-detail body meshes but keeping both centroids locked to an assumed perfect circular Keplerian orbit and keeping Beta locked to the line of syzygy. This gives a static setup in which to test the guaranteed-everywhere-lofting spin rate on the chosen face \# 4113 at different phases of that face from the syzygy plane. As expected from intuition about the radial component of tidal acceleration, two minima in this spin rate occur at $0^{\circ}$ and $180^{\circ}$ of phase. The minima are separated by about $1 \times 10^{-6} \mathrm{rad} / \mathrm{s}$ in value, the $0^{\circ}$ phase number being lower, also as expected given the asymmetry of tidal acceleration in such a system for which the ratio of mean primary radius to orbit size is not $\ll 1$. Hence the threshold for same side lofting is chosen to be $1 \times 10^{-6} \mathrm{rad} / \mathrm{s}$ lower than the $0.000641444 \mathrm{rad} / \mathrm{s}$ threshold chosen by the last paragraph for opposite side lofting. Fixing $0^{\circ}$ phase, the guaranteed-everywhere-lofting spin rate on face \# 4113 is also found to have a minimum at $\approx 2^{\circ}$ along the pole offset angle coordinate of parameter space. Varying Beta libration angle and slightly varying the in-plane orbit elements has minimal effect on first-lofting or guaranteed-everywhere-lofting spin rates.

All of this information is used to guide our choice of initial configuration conditions for simulation of the F2BP binary motion, and corresponding simulation of particle trajectories within the binary (as it follows that motion) starting from Alpha's surface, on the chosen lofting facet. For the latter simulation by 
the methods of section 3, at the initial time we place particles in a triangular grid over the triangular facet, with zero velocity relative to the moving surface. We displace them by a very small distance $(\approx 0.25 \mathrm{~m})$ along the face normal direction to avoid intersection with the body polyhedral mesh and immediate collision detection at the initial time. The particles are not given any physical size, and while a normalized mass is assigned to them within the RF3BP code, they essentially act as massless test particles because no influence on the underlying binary motion or on each other is modelled.

The results of batches of regolith particles thus propagated are sorted according to their final disposition (return impact onto Alpha, transfer impact onto Beta, escape from the system, and lingering in "orbit" at the maximum simulation duration). Those with each disposition are further sorted according to whether that outcome matches the expected outcome based on the initial energy of the particle's trajectory. The Jacobi energy function $J$ for a trajectory is time-varying in the full system. However for the earlier-mentioned R3BP and RSE3BP approximate systems, the potential through which a particle moves is time-invariant in the uniformly rotating barycentric frame, and hence the Jacobi function for a trajectory becomes a Jacobi integral. We can compute the integral's value at the L1 point for the R3BP, or at the analogue to the L1 point for the RSE3BP found in the rotating barycentric frame at the location $y=z=0$ and $x$ equal to the root of

$$
0=x-\frac{1-\nu}{(x+\nu)^{2}}-\nu(x-(1-\nu)) \frac{3}{2} \int_{\lambda}^{\infty} \frac{d u}{\left(u+\alpha^{2}\right) \Delta(u)}
$$

Here $\nu=m_{\beta} /\left(m_{\alpha}+m_{\beta}\right)$ again, and $\lambda=(x-(1-\nu))^{2}-\alpha^{2}$ with $\alpha$ being the longest semi-axis of the Beta ellipsoid, and $\Delta$ is as defined for this standard elliptic integral. Specifically, the Jacobi integral value for the RSE3BP when stationary at this RSE3BP L1-analogue point is

$$
J_{\mathrm{RSE} 3 \mathrm{BP}} \mathrm{L} 1=-\frac{1}{2} x_{\mathrm{L} 1}^{2}-\frac{1-\nu}{\left|x_{\mathrm{L} 1}+\nu\right|}-\nu \frac{3}{4} \int_{\lambda}^{\infty}\left(\frac{1}{\Delta(u)}-\frac{(x-(1-\nu))^{2}}{\left(u+\alpha^{2}\right) \Delta(u)}\right) d u .
$$

For each regolith particle propagated we compute from its initial conditions the initial RF3BP Jacobi function value using the following equation, with all variables with respect to the rotating barycentric frame:

$$
J_{\mathrm{RF} 3 \mathrm{BP}_{\mathrm{O}}}=\frac{1}{2} \dot{\boldsymbol{\rho}} \cdot \dot{\boldsymbol{\rho}}-\frac{1}{2}\left[\widetilde{\boldsymbol{\omega}_{\mathrm{orb}}} \boldsymbol{\rho}\right] \cdot\left[\widetilde{\boldsymbol{\omega}_{\mathrm{orb}}} \boldsymbol{\rho}\right]-u_{\alpha \beta}
$$

Herein the $u_{\alpha \beta}$ is evaluated with Eq. 14. Comparison between the $J_{\mathrm{RF} 3 \mathrm{BP}_{\mathrm{O}}}$ value and the $J_{\mathrm{R} 3 \mathrm{BP}} \mathrm{L}_{\mathrm{O}}$ or $J_{\mathrm{RSE} 3 \mathrm{BP}} \mathrm{L1}$ value for the approximate model equilibrium points gives an expected trajectory outcome. We can compare the actual propagated outcome against the expected one to do our further sorting, which characterizes the degree to which the time-varying nature of the full system's dynamics is important. We observe lofted particles going to transfer impact, "orbit", and escape outcomes only for the cases with the higher initial Alpha spin rate of $0.000651444 \mathrm{rad} / \mathrm{s}$, so all of this is relevant only to those cases.

When some particles go to dispositions other than return impact onto Alpha like this, it causes a problem with eventual draining of particles from the surface of Alpha in the probability-based simulation of the next subsection. It would also imply continuous erosion of material from the primary in the real binary system, which doesn't seem realistic at this stage in the system's evolution. Thus for results shown in this paper we employ the next subsection's methodology only with inputs derived from the results sets, for dynamic simulation of lofting particles on top of F2BP motion, matching our two cases with initial Alpha spin rate at the threshold spin rate values.

These inputs passed to the probability-based propagation are two key probability matrices (one for lofting from each side) and the means and standard deviations for velocities accompanying non-zero elements of those matrices. We divide the $X-Y$ plane (orbit plane) of the barycentric rotating frame into 36 angular sectors each $10^{\circ}$ wide converging at the location in that frame of the centroid of Alpha, and number these 1-36 counterclockwise, starting with the centerline of sector 1 lying on the line of syzygy opposite from Beta. We create the same type of "bins" in longitude relative to the rotating barycentric frame centered on Beta's centroid, aligned the same way, and number these 37-72. In post-processing a large ensemble of particles propagated using dynamic simulation, we find the longitude in this frame of the plane projection of the impact point of each particle trajectory, and find which bin that longitude falls within, for the appropriate body impacted. We also take the total time of flight, $t_{E}-t_{S}$, for the trajectory and obtain a time bin 
designation as

$$
\left\lfloor 18\left(\left\|\boldsymbol{\omega}_{\alpha}\right\|-\left\|\boldsymbol{\omega}_{\text {orb }}\right\|\right)\left(t_{E}-t_{S}\right) / \pi\right\rfloor+1 .
$$

The element of the probability matrix in the row matching the longitude bin number and column matching the time bin number is incremented by one. All particles impacting neither body, but instead escaping or orbiting at the maximum duration, we consider released to those final dispositions instantly. So we similarly count up these cases in the first column, in the last rows (with zeros to their right). The entire matrix is divided by the ensemble size to give probabilities of reaching each location in space and time, or outcome. For each impacting particle we find the velocity with respect to, and coordinated in, a local "bin-fixed" frame with $+X$ pointing outward along the bin of impact's centerline, $+Z$ aligned to the barycentric rotating frame's $+Z$ axis, and origin at the point of impact. All such impacting velocity vectors for particles reaching a bin are accumulated, and the mean and standard deviation is stored along with the matrix for use in the probabilistic algorithm described below. If applicable, for each escaping/orbiting particle we compute the mass-specific change to the particle's barycentric angular momentum between endpoints of its flight, average over all particles escaping/orbiting, and pass these vectors on for the below as well.

\subsection{Probabilistic simulation of regolith particles}

The preceding section's dynamic simulation of particles within a binary system treats the particles as test particles having mass but not exerting any influence on the translational or rotational motion of the binary components themselves, nor exerting any influence on each other's motion. This is justified by the fact that particles of realistic size and mass will generate forces and torques on the binary components that are so small compared to the forces and torques exerted by the binary components on each other as to be indistinguishable from numerical noise if included in the dynamic simulation. The scaling of the problem advocates against this approach. In addition, the accumulation of the binary components' responses to individual particle motions takes very long times to build up to observable changes, and propagation of the F2BP dynamics over such long time horizons is prohibitively expensive. Even if such computation were free, and despite the excellent symplectic and mean-error-reducing properties of the integration algorithm used, dynamic propagation to very long durations would entail eventual accumulation of numerical error which could confuse or overwhelm the accumulating particle motion effects on the binary.

However, it is of greater interest for questions of asteroid science to detail the slow time evolution of the binary system in response to the shed particles than it is to detail the fast particle trajectories themselves. Thus we outline in this subsection a probability-based method for propagating a simplified model of a binary system plus a collection of primary regolith particles. This model can be run forward for very long durations and much larger particle populations at a comparatively very low computational cost. As it uses the probability matrices and accompanying random variable parameters derived from post-processing a large set of particles propagated dynamic simulation, the probability-based method is grounded in the true full-detail dynamics of the system while computationally independent from them.

First we set up a particle state vector $\mathbf{x}$ with one element for each particle, the integer value of which indicates, along with some appropriate buffer variables, the particle's location in space and/or time or else that particle's final disposition. Admissible values for elements of $\mathbf{x}$ and their meanings are detailed in Table 1. At the initial time, $\mathbf{x}$ is set to uniformly distributed random integers in the range of 1-36, effectively spreading the equal-mass particles evenly around Alpha's equatorial longitude.

Note that for the rest of this paper we use the same notation conventions as before (vectors in bold, dyads and matrices in uppercase roman, tilde for the cross product matrix form). We also use a first-level right subscript to indicate the body the quantity applies to, a second-level right subscript to indicate a step or instant in time, a left subscript to indicate the frame a quantity is coordinated in, and a left superscript to denote the frame the quantity (such as velocity) is relative to. Any of these are omitted when not relevant or when the relationship holds independent of frame, etc. Also ${ }_{I}$ indicates the inertial barycentric frame, ${ }_{B}$ indicates the rotating barycentric frame, $\alpha$ indicates the Alpha-fixed frame or the Alpha body itself, $\beta$ indicates the Beta-fixed frame or the Beta body itself, and ${ }_{i}$ indicates the $i$ 'th particle.

Aside from the particle states, a set of binary states describing the system at any given time is set to initial values matching the binary model simulated as in the previous subsection. This set of states consists of the following: mass of each component, $m_{\alpha_{t}}$ and $m_{\beta_{t}}$, the inertia dyad of each component, ${ }_{\alpha} \mathrm{I}_{\alpha_{\mathrm{t}}}$ and ${ }_{\beta} \mathrm{I}_{\beta_{\mathrm{t}}}$, 
the angular velocity vector of each component, ${ }_{\alpha} \boldsymbol{\omega}_{\alpha_{t}}$ and ${ }_{\beta} \boldsymbol{\omega}_{\beta_{t}}$, the attitude rotation matrix mapping from the frame of each component to the inertial frame, ${ }_{I} \mathrm{R}_{\alpha_{\mathrm{t}}}$ and ${ }_{I} \mathrm{R}_{\beta_{\mathrm{t}}}$, and the barycentric position and velocity of each component center of mass, i.e. ${ }_{I} \mathbf{r}_{\alpha_{t}},{ }_{I}^{I} \mathbf{v}_{\alpha_{t}},{ }_{I} \mathbf{r}_{\beta_{t}}$, and ${ }_{I}^{I} \mathbf{v}_{\beta_{t}}$. In addition, the mean equatorial radius of Alpha, $d_{\alpha_{t}}$, is made variable to account for mass loss/addition from/to Alpha's equatorial region making Alpha slightly smaller/larger in size. We denote the time since the propagation start at discrete time step ${ }_{t}$ as $t_{t}$. The interval between discrete time steps is determined as the time needed for a point stationary on Alpha's surface to advance from one sector of longitude relative to the rotating barycentric frame, or one "longitude bin", to the next, that is

$$
t_{t+1}-t_{t}=\frac{\pi}{18\left(\left\|\omega_{\alpha_{t}}\right\|-\left\|\widetilde{\mathbf{r}_{t}}{ }_{I}^{I} \mathbf{v}_{t}\right\| /\left\|_{I} \mathbf{r}_{t}\right\|^{2}\right)}, \quad{ }_{I} \mathbf{r}_{t}={ }_{I} \mathbf{r}_{\beta_{t}}-{ }_{I} \mathbf{r}_{\alpha_{t}}, \quad{ }_{I}^{I} \mathbf{v}_{t}={ }_{I}^{I} \mathbf{v}_{\beta_{t}}-{ }_{I}^{I} \mathbf{v}_{\alpha_{t}} .
$$

This can be calculated at any time step, and likewise the angular momenta of the system components can be found at any step from the binary states:

$$
\begin{aligned}
& { }_{I} \mathbf{H}_{\alpha_{t}}={ }_{I} \mathrm{R}_{\alpha_{t} \alpha} \mathrm{I}_{\alpha_{t} \alpha} \boldsymbol{\omega}_{\alpha_{t}} \quad, \quad{ }_{I} \mathbf{H}_{\beta_{t}}={ }_{I} \mathrm{R}_{\beta_{t} \beta} \mathrm{I}_{\beta_{t} \beta} \boldsymbol{\omega}_{\beta_{t}}
\end{aligned}
$$

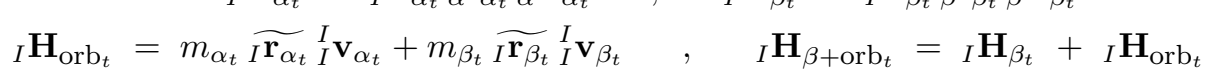

Before discussing the algorithm logic used to propagate from one time step to the next, we detail the changes to the binary states caused by lofting, gravitational interaction during flight, and then impact of a single particle of mass $m_{i}$. First for lofting. Since the particle is released with no initial velocity relative to Alpha's surface, no reaction between the particle and that surface takes place. The rotational state of Alpha (both attitude matrix and angular velocity vector) is preserved from just before lofting ("instant _") to just after lofting ("instant + "). However, Alpha's total mass is reduced by $m_{i}$ and the inertia dyad of Alpha is also changed, not only directly but by the re-shifting of the Alpha center of mass to account for the new body mass distribution. That centroid shift also slightly adjusts the barycentric position and velocity of the centroid. We have the following relations:

$$
\begin{aligned}
{ }_{\alpha} \boldsymbol{\omega}_{\alpha_{+}} & ={ }_{\alpha} \boldsymbol{\omega}_{\alpha_{-}} \\
{ }_{I} \mathrm{R}_{\alpha_{+}} & ={ }_{I} \mathrm{R}_{\alpha_{-}} \\
m_{\alpha_{+}} & =m_{\alpha_{-}}-m_{i} \\
{ }_{\alpha} \mathrm{I}_{\alpha_{+}} & ={ }_{\alpha} \mathrm{I}_{\alpha_{-}}+\left(\frac{m_{\alpha_{-}} m_{i}}{m_{\alpha_{-}}-m_{i}}\right) \widetilde{\boldsymbol{\rho}_{i_{-}}} \widetilde{\boldsymbol{\rho}_{i_{-}}} \\
{ }_{I} \mathbf{r}_{\alpha_{+}} & ={ }_{I} \mathbf{r}_{\alpha_{-}}-\left(\frac{m_{i}}{m_{\alpha_{-}}-m_{i}}\right){ }_{I} \mathrm{R}_{\alpha_{-}} \boldsymbol{\rho}_{i_{-}} \\
{ }_{I}^{I} \mathbf{v}_{\alpha_{+}} & ={ }_{I} \mathbf{v}_{\alpha_{-}}-\left(\frac{m_{i}}{m_{\alpha_{-}-m_{i}}}\right){ }_{I} \mathrm{R}_{\alpha_{-}} \widetilde{\boldsymbol{\omega}_{\alpha_{-}}}{ }_{\alpha} \boldsymbol{\rho}_{i_{-}}
\end{aligned}
$$

Here the quantity ${ }_{\alpha} \boldsymbol{\rho}_{i_{-}}$is the relative position vector from the centroid of Alpha to the position of the particle $i$ at the instant before lofting, expressed in the Alpha frame at the instant before lofting, prior to the centroid shift involved. The location on Alpha's equator where we determine the lofting will occur gives this vector. In our model we choose this equatorial location to be either the center of the rotating-barycentricframe-relative longitude bin 1 (i.e. on the line of syzygy opposite from Beta, or at the anti-Beta point on Alpha) or else the center of the similar longitude bin 19 (on the line of syzygy on the same side as Beta, or at the sub-Beta point on Alpha). Note, for later use in defining the starting point of the trajectory followed by each lofted particle,

$$
\begin{aligned}
& { }_{I} \mathbf{r}_{i_{+}}={ }_{I} \mathbf{r}_{\alpha_{-}}+{ }_{I} \mathrm{R}_{\alpha_{-}} \alpha \boldsymbol{\rho}_{i_{-}} \\
& { }_{I}^{I} \mathbf{v}_{i_{+}}={ }_{I}^{I} \mathbf{v}_{\alpha_{-}}+{ }_{I} \mathrm{R}_{\alpha_{-}} \widehat{\alpha \boldsymbol{\omega}_{\alpha_{-}}}{ }_{\alpha} \boldsymbol{\rho}_{i_{-}} .
\end{aligned}
$$

Next for the set of changes in the binary states that occur from just before to just after impact of the particle onto either body. Here we show the relations for a return impact back onto Alpha rather than a transfer impact onto Beta. However, all of the relations are identical in the latter case except for replacing 
the subscripts $\alpha$ with $\beta$, and using the new quantities thus denoted for Beta. Upon particle impact, the particle mass is added onto the target body with an accompanying direct change to the body inertia dyad plus an indirect change to the same due to body centroid re-shifting. Again, such a centroid shift adjusts the barycentric position and velocity of the centroid. The instantaneous attitude of the body may be assumed unchanged across the impact, but because the incoming particle has velocity relative to the surface just before impact, the body reacts to that with a change in angular velocity (and an extra additive change in inertial barycentric velocity of the body centroid too). Assume for now that the other, final endpoint of the trajectory followed by each lofted but then impacting particle is well-defined as ${ }_{I} \mathbf{r}_{i_{-}}$and ${ }_{I}^{I} \mathbf{v}_{i_{-}}$. Then we have that

$$
{ }_{\alpha} \boldsymbol{\rho}_{i_{-}}={ }_{I} \mathrm{R}_{\alpha_{-}}^{T}\left({ }_{I} \mathbf{r}_{i_{-}}-{ }_{I} \mathbf{r}_{\alpha_{-}}\right)
$$

Then we have the following relations for the binary state changes across an impact:

$$
\begin{aligned}
& { }_{I} \mathrm{R}_{\alpha_{+}}={ }_{I} \mathrm{R}_{\alpha_{-}} \\
& m_{\alpha_{+}}=m_{\alpha_{-}}+m_{i} \\
& { }_{\alpha} \mathrm{I}_{\alpha_{+}}={ }_{\alpha} \mathrm{I}_{\alpha_{-}}-\left(\frac{m_{\alpha_{-}} m_{i}}{m_{\alpha_{-}}+m_{i}}\right) \widetilde{\alpha \boldsymbol{\rho}_{i_{-}}} \widetilde{\alpha \boldsymbol{\rho}_{i_{-}}} \\
& { }_{I} \mathbf{r}_{\alpha_{+}}={ }_{I} \mathbf{r}_{\alpha_{-}}+\left(\frac{m_{i}}{m_{\alpha_{-}}+m_{i}}\right){ }_{I} \mathrm{R}_{\alpha_{-} \alpha} \boldsymbol{\rho}_{i_{-}} \\
& { }_{I}^{I} \mathbf{v}_{\alpha_{+}}=\left(\frac{m_{\alpha_{-}}}{m_{\alpha_{-}}+m_{i}}\right){ }_{I}^{I} \mathbf{v}_{\alpha_{-}}+\left(\frac{m_{i}}{m_{\alpha_{-}}+m_{i}}\right){ }_{I}^{I} \mathbf{v}_{i_{-}} \\
& { }_{\alpha} \boldsymbol{\omega}_{\alpha_{+}}=\left[{ }_{\alpha} \mathrm{I}_{\alpha_{-}}-\left(\frac{m_{\alpha_{-}} m_{i}}{m_{\alpha_{-}}+m_{i}}\right) \widetilde{\alpha \boldsymbol{\rho}_{i_{-}}} \widetilde{\alpha \boldsymbol{\rho}_{i_{-}}}\right]^{-1} \text {. } \\
& \left({ }_{\alpha} \mathrm{I}_{\alpha_{-} \alpha} \boldsymbol{\omega}_{\alpha_{-}}+\left(\frac{m_{\alpha_{-}} m_{i}}{m_{\alpha_{-}}+m_{i}}\right){ }_{I} \mathrm{R}_{\alpha_{-}}^{T}\left[{ }_{I} \mathbf{r}_{i_{-}}-{ }_{I} \mathbf{r}_{\alpha_{-}}\right]\left[{ }_{I}^{I} \mathbf{v}_{i_{-}}-{ }_{I}^{I} \mathbf{v}_{\alpha_{-}}\right]\right)
\end{aligned}
$$

There are also small changes to the binary states with the assumed reaction of the binary system orbit to balance modification of a particle's inertial barycentric angular momentum while it is in flight. This angular momentum change occurs due to gravitational interaction between the particle and the full mass distributions of the binary components. Again let the particle's trajectory be defined, with starting point denoted here as $\left\{{ }_{I} \mathbf{r}_{i_{S}},{ }_{I}^{I} \mathbf{v}_{i_{S}}\right\}$ and ending point denoted here as $\left\{{ }_{I} \mathbf{r}_{i_{E}},{ }_{I}^{I} \mathbf{v}_{i_{E}}\right\}$. Then across the bit of interaction during each of the $p$ discrete time intervals (assumed roughly equal in length) covered by the particle's trajectory, we have

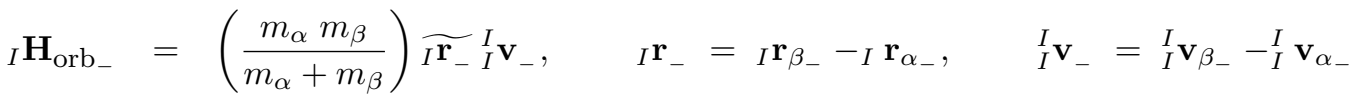

$$
\begin{aligned}
& { }_{I} \mathbf{H}_{\mathrm{orb}_{+}}={ }_{I} \mathbf{H}_{\mathrm{Orb}_{-}}-\frac{m_{i}}{p}\left(\widetilde{{ }_{I} \mathbf{r}_{i_{E}}}{ }_{I}^{I} \mathbf{v}_{i_{E}}-\widetilde{{ }_{I} \mathbf{r}_{i_{S}}}{ }_{I} \mathbf{v}_{i_{S}}\right) \\
& e=\left({ }_{I} \widetilde{\mathbf{H}_{\mathrm{Orb}_{-}}}{ }_{I} \mathbf{H}_{\mathrm{Orb}_{+}}\right) /\left\|\widetilde{\mathbf{H}_{\mathrm{Orb}_{-}}} \mathbf{H}_{\mathrm{Orb}_{+}}\right\| \\
& \theta=\operatorname{acos}\left(\frac{{ }_{I} \mathbf{H}_{\mathrm{Orb}_{-}}}{\left\|{ }_{I} \mathbf{H}_{\mathrm{Orb}_{-}}\right\|} \cdot \frac{{ }_{I} \mathbf{H}_{\mathrm{Orb}_{+}}}{\left\|{ }_{I} \mathbf{H}_{\mathrm{Orb}_{+}}\right\|}\right) \\
& R=\operatorname{expm}[\widetilde{e} \theta] \\
& k=\left[\begin{array}{l}
0 \\
0 \\
1
\end{array}\right] \cdot \frac{\mathbf{H}_{\mathrm{orb}_{+}}}{R_{I} \mathbf{H}_{\mathrm{orb}_{-}}+\delta} \\
& { }_{I} \mathbf{r}_{\alpha_{+}}=\left(\frac{-m_{\beta}}{m_{\alpha}+m_{\beta}}\right) k^{2} R_{I} \mathbf{r}_{-} \quad{ }_{I} \mathbf{r}_{\beta_{+}}=\left(\frac{m_{\alpha}}{m_{\alpha}+m_{\beta}}\right) k^{2} R_{I} \mathbf{r}_{-} \\
& { }_{I} \mathbf{v}_{\alpha_{+}}=\left(\frac{-m_{\beta}}{m_{\alpha}+m_{\beta}}\right) \frac{1}{k} R_{I}^{I} \mathbf{v}_{-} \quad{ }_{I} \mathbf{v}_{\beta_{+}}=\left(\frac{m_{\alpha}}{m_{\alpha}+m_{\beta}}\right) \frac{1}{k} R_{I}^{I} \mathbf{v}_{-}
\end{aligned}
$$


Herein $\delta$ is a very small constant close to machine precision to prevent singularity, expm $[\cdot]$ is the matrix exponential operation, and there are no time subscripts on the component masses because they do not change across this time-step's bit of the interaction. Note that large $p$ for longer flight times can be problematic in that very large $p$ spreads out the change across many time steps until the change improperly numerically disappears given an override needed to avoid numerical error. When the orbit angular momentum change of Eq. 39 is segmented into many equal portions applied during each of the time steps for which the particle is in the "in-flight" state, each portion may be too small, such that ${ }_{I} \mathbf{H}_{\mathrm{orb}}$ and ${ }_{I} \mathbf{H}_{\mathrm{Orb}_{+}}$are so close in direction that the dot product inside of the acos in Eq. 41 is nearly unity. In fact, numerical error may result in the dot product being slightly greater than unity, producing imaginary numbers which break the propagation. Therefore an override is introduced to force the dot product to be $\leq 1$, and thus $\theta=0$, in such rare cases.

As for the determination of the final endpoint of a particle's trajectory, and how much later in time that comes than the initial endpoint of the trajectory, that information is derived from the probability matrices from the earlier post-processing of the particle dynamic simulation output. Recall that each matrix has rows corresponding to the particle state values 1-74, i.e. corresponding to bins of longitude on Alpha, then bins of longitude on Beta, then the escaping and lingering in orbit final dispositions (see Table 1). Also recall the columns correspond to the number of intervals in time covered by the trajectory. The location in time, body reached, and equatorial position on that body (centerlines of longitude bins are used) of a trajectory's final endpoint is randomly selected in roulette wheel fashion using the nonzero probabilities in the probability matrix. This gives the needed inertial position vector ${ }_{I} \mathbf{r}_{E}={ }_{I} \mathbf{r}_{i_{-}}$(the latter with respect to the impact equations), computed at point where it must be used given then-current binary state values. Then the particle's velocity in the bin-fixed frame attached to the longitude bin reached by the particle is generated from a normal random variable draw, using the mean and standard deviation corresponding to that bin, as read in along with the matrices. This velocity is converted into the needed inertial particle velocity ${ }_{I}^{I} \mathbf{v}_{i_{E}}={ }_{I}^{I} \mathbf{v}_{i_{-}}$(the latter with respect to the impact equations). The conversion is made at the point of use for the inertial velocity, given then-current binary state values.

Finally note that the progression of time from one step to the next itself also requires an update of the binary states. Since the mutual orbit is nearly circular, we can approximate the update for the orbit states with a simple rotation about the orbit normal. We can also approximate the updates for the body attitudes with simple rotations about each body's instantaneous angular velocity direction. For Alpha, this simple rotation must also account for any small, constant angular acceleration of Alpha, $\boldsymbol{\alpha}_{\alpha}$, included in the model to represent YORP or torque from any source external to the binary. Assuming alignment between the angular acceleration and the spin vector in the Alpha-fixed frame we have

$$
\begin{aligned}
& { }_{I} \mathrm{R}_{\alpha_{t+1}}={ }_{I} \mathrm{R}_{\alpha_{t}} \operatorname{expm}\left[\widetilde{\boldsymbol{\omega}_{\alpha_{t}}} \frac{\left\|\boldsymbol{\alpha}_{\alpha}\right\|\left(t_{t+1}-t_{t}\right)^{2}}{2\left\|\boldsymbol{\omega}_{\alpha_{t}}\right\|}+\widetilde{\boldsymbol{\omega}_{\alpha_{t}}}\left(t_{t+1}-t_{t}\right)\right]^{T} \\
& { }_{I} \mathrm{R}_{\beta_{t+1}}={ }_{I} \mathrm{R}_{\beta_{t}} \operatorname{expm}\left[\widetilde{\boldsymbol{\omega}_{\beta_{t}}}\left(t_{t+1}-t_{t}\right)\right]^{T} \\
& { }_{\alpha} \boldsymbol{\omega}_{\alpha_{t+1}}={ }_{\alpha} \boldsymbol{\omega}_{\alpha_{t}}\left(1+\frac{\left\|\boldsymbol{\alpha}_{\alpha}\right\|}{\left\|\boldsymbol{\omega}_{\alpha_{t}}\right\|}\left(t_{t+1}-t_{t}\right)\right) \\
& R_{\text {orb }}=\operatorname{expm}\left[\frac{\widetilde{{ }_{I} \mathbf{r}_{t}^{I} \mathbf{v}_{t}}}{\left\|\widetilde{\mathbf{r}}_{t}{ }_{I} \mathbf{v}_{t}\right\|} \sqrt{G\left(m_{\alpha_{t}}+m_{\beta_{t}}\right) /\left\|_{I} \mathbf{r}_{t}\right\|^{3}}\left(t_{t+1}-t_{t}\right)\right]
\end{aligned}
$$

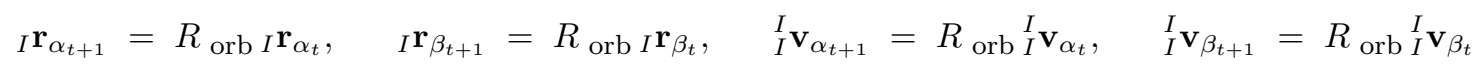

Now that all of the building blocks for the algorithm have been detailed, we describe the algorithm logic used to propagate from one time step to the next. Recall that initially all particle states $\mathbf{x}_{i}$ have values between 1 and 36 representing the particle lying on Alpha's surface in the corresponding longitude bin. First the binary states at a new time step ${ }_{t+1}$ are set equal to those at the previous step ${ }_{t}$. Then we loop through all particles to determine any motion/changes for each particle and the accompanying changes to the new time step binary states based on what happens to that particle.

If $1<\mathbf{x}_{i_{t}}<19$ or $19<\mathbf{x}_{i_{t}}<37$ then the particle was not previously at a lofting location on Alpha's surface, and remains on the surface but is carried around to the next longitude bin (going counterclockwise) 
with Alpha's rotation relative to the rotating barycentric frame. So $\mathbf{x}_{i_{t+1}}=\mathbf{x}_{i_{t}}+1$, or in the case of $\mathbf{x}_{i_{t}}=36$, we wrap back with $\mathbf{x}_{i_{t+1}}=1$. If however $\mathbf{x}_{i_{t}}=\{1,19\}$ then the particle was previously at a lofting location and so we compare $\left\|\boldsymbol{\omega}_{\alpha_{t}}\right\|$ with the appropriate threshold rate below which lofting on the opposite or same side from Beta turns off, and compare the value of $d_{\alpha_{t}}$ with the threshold mean Alpha radius below which lofting turns off everywhere. If either quantity is too small, $\mathbf{x}_{i}$ is again incremented by 1 to represent remaining on the rotating surface, but if both quantities are sufficiently large the particle lofts. In the latter case the corresponding probability matrix is used to randomly assign the particle to go to a bin in longitude vs. flight-time space.

If the column designation $y$ of the bin in that space is 1 , the particle is considered to go to the specified impact location immediately or start it's escape or orbiting path immediately, so $\mathbf{x}_{i_{t+1}}$ is assigned the value of the row of the bin in that space. If this value is between 1 and 36 inclusive, then changes to the current (indexed by ${ }_{t+1}$ ) binary system states are made matching lofting (Eqs. 23-28), flight which covers only one time interval (Eqs. 38-45 with $p=1$ ), and return impact onto Alpha (Eqs. 31-37). If the value is between 37 and 72 inclusive, then changes to the current binary states are made matching lofting, one time interval of flight, and impact onto Beta (using the same equations, respectively). If the value is 73 for escape or 74 for orbiting, changes to current binary states are made just for lofting (Eqs. 23-28) and for the entire escape or orbiting path at once (Eqs. 38-45 but replacing Eq. 39 with ${ }_{I} \mathbf{H}_{\mathrm{orb}_{+}}={ }_{I} \mathbf{H}_{\mathrm{orb}_{-}}-m_{i}{ }_{I} \Delta \mathbf{H}_{i}$ where ${ }_{I} \Delta \mathbf{H}_{i}$ is the average mass-specific angular momentum change to an escaping/orbiting particle).

If the column designation $y$ of the bin in longitude vs. flight-time space that the particle is assigned to after lofting is instead $>1$, then $\mathbf{x}_{i_{t+1}}=10000-y+1$. Thus this particle state is set to be 10000 less

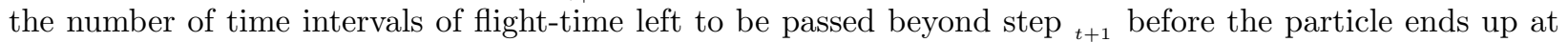
the specified impact location matching the row of the bin in the longitude vs. flight-time space. The row designation $x$ of the bin is placed in a buffer variable for retrieval later when the impact time arrives, and $y$ is also so buffered. In the present time step, changes are made to the current ${ }_{t+1}$ binary states for lofting (Eqs. 23-28) and for just the first segmented portion of the interaction along the trajectory (Eqs. 38-45 with $p$ equal to the buffered $y$ ).

Back up at the level of testing the particle state's previous value $\mathbf{x}_{i_{t}}$, if $74<\mathbf{x}_{i_{t}}<10000$ then the particle was previously lofted and was awaiting impact as of step ${ }_{t}$. We use $\mathbf{x}_{i_{t+1}}=\mathbf{x}_{i_{t}}+1$ to move the particle toward impact in time and make the changes to the binary states for another segmented portion of the particle's interaction along the trajectory (Eqs. 38-45 with $p$ equal to the $y$ buffered for this particle). If after so incrementing $\mathbf{x}_{i}$ we then have $\mathbf{x}_{i_{t+1}}=10000$, then the particle has moved to impact in covering the current time interval to reach step ${ }_{t+1}$. Thus we reassign $\mathbf{x}_{i_{t+1}}=x$ ( $x$ being buffered for this particle) to indicate where the particle impacts and additionally apply the changes to binary states matching the impact (Eqs. 31-37).

If $36<\mathbf{x}_{i_{t}}<75$ then the the particle state value is kept the same, i.e. $\mathbf{x}_{i_{t+1}}=\mathbf{x}_{i_{t}}$, because no further details of motion is included in our present modelling of the system for particles that have already transfer impacted onto Beta or already embarked upon escaping or orbiting paths.

Once completing the loop through all particle states to determine changes to particle disposition and matching binary state changes, the time $t$ is increased by one interval to get $t_{t+1}$ and the regular updates to the current binary states for time evolution of the binary by itself are applied (Eqs. 46-50, reconsidering the binary states before use of these equations as if being for the previous time index $\left.{ }_{t}\right)$. Also, $d_{\alpha_{t+1}}$ is found anew from $m_{\alpha_{t+1}}$. Then all of the above may be iterated for the next time step.

Before doing so, however, a few additional tasks are optionally performed for better fidelity and improved running time. First, because the spin rate of Alpha may have changed over the last time step in response to accumulated effects of particles return impacting onto Alpha, and because the mutual orbit rate will have changed as well, the length of a time interval according to the definition of Eq. 21 may have increased or decreased. This time interval re-scaling may require shifting of the value of any particle state with value at this point in the algorithm in the range of $100<\mathbf{x}_{i}<9999$ either higher (for increasing interval length) or lower (for decreasing interval length). Recall that $10000-\mathbf{x}_{i}$, for $\mathbf{x}_{i}$ in this range, represents the number of time intervals past the current time yet to pass before the particle will impact. Multiplying the ratio of the old interval length to the new one by this $10000-\mathbf{x}_{i}$ and taking the fractional remainder thereof gives a value for a uniform random draw in the interval 0,1 to be compared with to determine incrementing/decrementing of $\mathbf{x}_{i}$.

Finally, it is observed that lofting, flight, and return impact often occur in rapid succession in short 
transient lofting episodes, separated by long time spans during which the primary is slowly spun back up by the YORP angular acceleration applied to it. Eventually the lower of the lofting spin rate thresholds is encountered again, and the cycle is repeated. Progressing through natural time steps by all of the above algorithm during the long spin-up periods would be computationally wasteful, as it would explode simulation run time for long durations without revealing further dynamically interesting behavior. Thus, at the point just before looping back to go through all operations for the next natural time step, if a detection buffer indicates that for the last ten natural time steps there was both no lofting activity and no particle state values indicating a particle was aloft, an extra time step to skip most of the spin-up period is added in. This skip-over step has length $\Delta t$ equal to $95 \%$ of the difference between the norm of ${ }_{\alpha} \boldsymbol{\omega}_{\alpha_{t}}$ and the lower spin rate threshold value, divided by the norm of the constant angular acceleration ${ }_{\alpha} \boldsymbol{\alpha}_{\alpha}$. Since nothing is aloft, no particle state adjustment as in the last paragraph is needed with this jump in time. Instead, all particle states in the range $1 \leq \mathbf{x}_{i_{t}} \leq 36$ are moved to account for the rotation of Alpha during the skip-over step, i.e. the number of bins to move through, $q$, and new particle state values are given by

$$
q=\operatorname{round}\left\{\frac{18}{\pi}\left(\frac{1}{2}\left\|\boldsymbol{\alpha}_{\alpha}\right\| \Delta t^{2}+\left\|\boldsymbol{\omega}_{\alpha_{t}}\right\| \Delta t-\sqrt{G\left(m_{\alpha_{t}}+m_{\beta_{t}}\right) /\left\|\mathbf{r}_{t}\right\|^{3}} \Delta t\right)\right\}, \quad \mathbf{x}_{i_{t+1}}=q-36\lfloor q / 36\rfloor
$$

Before updating the system's binary states across the skip-over time step, we make the reasonable assumption that damping of non-principal-axis rotation in the primary occurs on a much faster timescale than the primary's spin-up by YORP, and perform a scaling and realignment to rotation about the body principal axis frame's $Z$-axis. This frame is recomputed for Alpha's current mass distribution, with corresponding shifts made to ${ }_{\alpha} \mathrm{I}_{\alpha_{t}}$ and ${ }_{I} \mathrm{R}_{\alpha_{t}}$, and then we use

$$
{ }_{\alpha} \boldsymbol{\omega}_{\alpha_{t}}=\left[\begin{array}{lll}
0, & 0, \frac{\left\|_{I} \mathrm{R}_{\alpha_{t} \alpha} \mathrm{I}_{\alpha_{t} \alpha} \boldsymbol{\omega}_{\alpha_{t}}\right\|}{\alpha \mathrm{I}_{\alpha_{t}}(3,3)}
\end{array}\right]^{T}
$$

The binary states are then updated using Eqs. $46-50$ with $t_{t+1}-t_{t}$ now replaced by $\Delta t$, and the time $t$ is increased by $\Delta t$ to get $t_{t+1}$. Then the regular time interval length is recomputed to account for the changes to the Alpha spin and mutual orbit rates, and system angular momenta are found again from current binary states before resetting the gradual spin-up period detection buffer and proceeding with the next natural time step.

\section{Results for Regolith Particle Motion and Binary System Behavior}

For several batches of RF3BP particle dynamic simulation output generated, the final dispositions resolved for the particles are broken down as shown in Table 2. As can be seen, only for cases with the higher initial Alpha spin rate of $0.000651444 \mathrm{rad} / \mathrm{s}$ do we observe lofted particles going to outcomes of transfer impact, still lingering in "orbit" (at a two-week cutoff duration), or escape. For all such particles the expected outcome by comparison of the initial Jacobi function value with the L1-analogue equilibrium point Jacobi function value, is instead return impact, which we would have without including detailed modelling of the time-varying dynamics for this system. However, dropping the initial Alpha spin rate by only $0.10-0.11 \times 10^{-5} \mathrm{rad} / \mathrm{s}$ produces total return impact probability of unity for the cases of $2.5^{\circ}$ offset angle between the orbit normal and Alpha's spin pole, for both same-side lofting position and opposite-side lofting position.

First we show results for a "nominal" probability-based simulation using the probability matrices from these last two cases, and using our best estimate for KW4 Alpha's YORP acceleration $\left(3 \times 10^{-11} \mathrm{rad} / \mathrm{s} /\right.$ year, or $\approx 9.5129 \times 10^{-19} \mathrm{rad} / \mathrm{s}^{2}$, per Scheeres et al. (2006)). The total mass of regolith or surface material free to move or be lofted is an uncertain physical parameter of the system's primary, so we nominally set this to 10 million metric tons, only about $0.43 \%$ of Alpha's total estimated mass. How this is discretized into particles with specific masses is also a free parameter. We nominally use 5000 particles of equal mass, though different choices for all of these parameters are explored below. The results of a nominal model simulation, extending to a duration of about 2792 years, are shown in Figure 2.

As previously mentioned, it is seen that lofting from the same side of Alpha as Beta, flight, and return impact occur quickly within short transient lofting episodes, separated by long time spans during which the primary is slowly spun back up by the YORP angular acceleration applied to it. Eventually the lower of the lofting spin rate thresholds is encountered again, and the cycle is repeated. The greater the depression of 
the Alpha spin rate during any single lofting episode, the longer the time needed for spin-up to lofting again and the longer the following separation time until the next lofting episode becomes. This separation time is seen to follow an exponential distribution, with mean of about 16.6 years for the nominal model given its applied angular acceleration on Alpha.

With the time axis scaled as it is in Figure 2, the transient episodes show up as "spikes" in the plotted quantities. Note the vertical scale in panel (b) of Figure 2 in comparison to the vertical scales on panels (a) and (c) mean that the angular momentum of Beta experiences only miniscule changes in the fashion of a random walk, and only in the $X$ - and $Y$-components. Therefore panel (c) really shows exclusively change to the orbit angular momentum, in the form of a steady increase in the main $Z$-component. The random walk changes in the $X$ - and $Y$ - components for Alpha and the orbit-plus-Beta mirror each other according to total angular momentum conservation for the system in those components. The external angular momentum injected to the system through the angular acceleration applied to Alpha is visibly transferred into expansion of the orbit, as was expected.

In addition to the mean separation time between lofting, there are other metrics for the level of particle lofting activity in the system. The duration covered by a simulation given a fixed number of time steps taken is one of these, as more time steps are used in propagating through lofting episodes than to cover even very long spin-up periods, so a shorter duration represents greater activity. In addition, taking the time-integral average of the mass lost from Alpha (as shown in panel (d) of Figure 2) gives an average amount of mass aloft, reflecting not only the frequency but individual length of lofting episodes. The total accumulated amount of mass lofted during a simulation divided by the total duration also gives an average mass lofting rate. However, the episodic nature of particle motion, when at low applied Alpha angular acceleration, means these activity metrics should not be misinterpreted to mean certain amounts of material are always hovering above the surface near the equator, or to mean material is continuously being levitated there.

However, it is of interest to examine what happens when the qualification above of low (i.e. YORPlevel) angular acceleration no longer applies. This is the hypothetical case we might have with a similarlyproportioned system of extremely small body sizes and extremely varied primary surface Albedo, or for a more reasonably sized typical asynchronous binary whose primary is artificially spun up using propulsive devices attached to it, for some unknown motivation. Similarly, it is interesting to explore various values for our other model parameters of particle mass, number of particles, and their product of total mass available to loft. Table 3 shows the degree and frequency of lofting activity within the system, represented by the metrics discussed above, versus the applied angular acceleration and these three material parameters. In turn, we vary the total number of particles and thus total regolith mass while holding particle size constant, vary the particle size and number of particles inversely while holding total regolith mass constant, and vary particle size and thus total regolith mass while holding the number of particles constant. Some of the trends within the table are also summarized in Figures 3-6.

It is seen that for the same number of particles but increasing total regolith mass, because the same number of particles come around to loft on the Beta-facing side when the threshold rate is encountered, but each one of those particles is more massive, the depression of Alpha spin rate during the lofting episode is greater, increasing the mean time between episodes and increasing the duration reached. However the timeaveraged amount of material aloft and mass lofting rate hold roughly constant, meaning larger mass motion occurring less frequently is the same as smaller mass motion occurring more frequently. The exception comes with moving to the largest total regolith mass case, also our nominal simulation case with output shown in Figure 2. The reason for this sharp increase is unclear, as the nature of the angular momenta behavior remains the same for these other cases as shown in the plots. Holding the total regolith mass constant, fewer but larger particles or pieces to loft also tends to increase the time between lofting episodes and duration, though less strongly. This is especially true for the lowest particle counts, with which we have the coarsest mass discretization and most uneven initial mass distribution around Alpha's equator. Since increasing the number of particles each having a fixed mass seems to extend the episode separation times and the duration, overall the effect of having more mass available to launch overpowers the effects of coarser mass discretization. Regardless, the time-averaged mass aloft and mass lofting rate hold roughly constant across all parameter changes for the lofting material. That is not the case for varying the applied angular acceleration. With low values for this, characteristic of YORP torque on real binary system primaries, the average mass aloft and average mass lofting rate smoothly vary proportionally with the applied angular acceleration.

Interestingly, that proportionality goes away once, for the currently examined KW4 system's body 
properties, the angular acceleration increases above about $\left\|\boldsymbol{\alpha}_{\alpha}\right\| \approx 10^{-14} \mathrm{rad} / \mathrm{s}^{2}$ (see Figure 3). Above this value the angular acceleration is large enough to eventually overwhelm the damping effect of sameside particle lofting on the Alpha spin rate after a certain amount of time has passed. Then a major shift occurs to non-episodic nearly continuous lofting with sustained mass loss from Alpha and sustained changes to it's moments of inertia. As the continuous lofting from the same side as Beta actually balances the applied acceleration less effectively than the prior intermittent lofting, the Alpha spin rate eventually increases through the $1 \times 10^{-6} \mathrm{rad} / \mathrm{s}$ separation to the threshold for lofting from the side opposite Beta. Once that particle motion also occurs, the now very large amount of replenishing material aloft (see Table 3) should begin to truly represent a sustained low-lying debris cloud or surface-grazing ring of debris around the equator. A second major shift in the system behavior also occurs at this point in time, beyond another large reduction in Alpha's mass and polar moment of inertia as the lofting region on the opposite side also becomes bare of material. In particular, the gravitational interaction of particles lofted from the opposite side with the binary components tends to drive the angular momentum transfer in the opposite direction as gravitational interaction between particles lofted from the same side with the binary components, and the former effect overpowers the latter in magnitude. Hence the spin rate of Alpha and the Alpha angular momentum rapidly increase while the orbit rapidly decays after this time. All of this behavior is shown in Figure 7 for the most extreme acceleration case in Table 3 having $\left\|\boldsymbol{\alpha}_{\alpha}\right\|=1.5 \times 10^{-13} \mathrm{rad} / \mathrm{s}^{2}$. Note the two major shifts discussed above occurring at approximately $1.0 \times 10^{6}$ and $1.31 \times 10^{6}$ seconds.

Eventually, the Alpha spin rate will increase to a point where the probability matrices derived from the previous full-detail dynamics simulation of particles in the binary no longer apply, after which the output can no longer be trusted. Instead the probability matrices and matching impact velocity information should be replaced with new versions obtained from such RF3BP dynamic simulation starting at higher initial Alpha spin rates. We infer from Table 2 that using the probability matrices for such higher Alpha spin rate cases will produce steady bleeding away of lofted material from the vicinity of Alpha to the "sinks" of impact with Beta and escape from the system. While this is an interesting new regime of behavior to study, we do not try to explore it further by repeatedly shifting to new matrices and new data grounded in new full dynamic simulation of particles. The reader is reminded that all of this applies only for hypothetical very large externally applied torque on the primary, not found naturally in binary asteroid systems.

\section{Implications for Binary System Morphology and Evolution}

For both the nominal case, with angular momentum behavior as shown in the first three panels of Figure 2, and all other cases also having $\left\|\boldsymbol{\alpha}_{\alpha}\right\| \ll 10^{-14} \mathrm{rad} / \mathrm{s}^{2}$ and hence having similar-looking plots of angular momentum changes, the slope of the linear fit to the combined orbit plus Beta angular momentum matches that expected. That is, the slope of the linear fit to the numerical output for this quantity, divided by the primary's initial or time-averaged moment of inertia about the $Z$-axis, recovers the angular acceleration applied to the primary about the $Z$-axis to within a few percent. Therefore the angular momentum injected to the system is seen to be fully transferred to the orbit, to which Beta is also synchronously locked onaverage. The results imply that for any given time, it is true that $\left\|\dot{\mathbf{H}}_{\beta+\text { orb }}\right\|={ }_{\alpha} \mathrm{I}_{\alpha_{\mathrm{ZZZ}}}\left\|\boldsymbol{\alpha}_{\alpha}\right\|$. Here the ${ }_{\alpha} \mathrm{I}_{\alpha_{\mathrm{ZZ}}}$ is the $Z$ - principal axis moment of inertia component of the inertia dyad for the primary expressed in its own frame. We can use this relation within a simple equation for the rate of change in the mutual orbit semi-major axis, $a$, as a function of the YORP angular acceleration acting on the primary body, accounting for the presence of the synchronous secondary:

$$
\dot{a}=\frac{2{ }_{\alpha} \mathrm{I}_{\alpha_{\mathrm{ZZ}}}\left\|\boldsymbol{\alpha}_{\alpha}\right\|}{\left(\frac{m_{\alpha} m_{\beta}}{m_{\alpha}+m_{\beta}}-3{ }_{\beta} \mathrm{I}_{\beta_{\mathrm{ZZ}}} / \mathrm{a}^{2}\right) \sqrt{\frac{G\left(m_{\alpha}+m_{\beta}\right)}{a}}}
$$

For our nominal results case, the slope of the orbit plus Beta angular momentum is $0.4459 \mathrm{~kg} \mathrm{~m} \mathrm{~m}^{2} / \mathrm{s}^{2}$, and when used with the KW4 system parameters in the above this translates to a current $0.881 \mathrm{~m} / \mathrm{kyr}$ rate of semi-major axis growth for the KW4 system. This orbit expansion is due to the surface particle motion angular momentum transfer mechanism alone and is several times faster than the orbit expansion resulting from the usual solid-body tidal evolution also present in the system. We do not in this work address any orbit evolution effects of asymmetric thermal absorption and reradiation to/from the secondary body, or so-called "BYORP", which may also be present in addition to the above two sources of orbit evolution. 
Integrating Eq. 53, we can obtain an expression for the time period required to increase the orbit size by some ratio $\zeta$ over an initial orbit size $a_{o}$, given just the dominating system evolution effect from angular momentum transfer due to lofting particle motion:

$$
T_{a_{o} \mapsto \zeta a_{o}}=\left[\sqrt{\zeta}-1-\frac{{ }_{\beta} \mathrm{I}_{\beta_{\mathbf{Z Z}}}}{m a_{o}^{2}}+\frac{{ }_{\beta} \mathrm{I}_{\beta_{\mathrm{ZZ}}}}{m \zeta^{\frac{3}{2}} a_{o}^{2}}\right] \frac{m \sqrt{G\left(m_{\alpha}+m_{\beta}\right) a_{o}}}{{ }_{\alpha} \mathrm{I}_{\alpha_{\mathrm{ZZ}}}\left\|\boldsymbol{\alpha}_{\alpha}\right\|}
$$

For our KW4 model, this gives the time since the system was at half it's present orbit size, time to double orbit size over the present, and time to double the orbit size again after that, of $1.70 \mathrm{Myr}, 2.46 \mathrm{Myr}$, and 3.50 Myr, respectively. Taking the distance covered by each such stage of orbit growth and dividing it by the time required gives average rates during those stages of $0.746 \mathrm{~m} / \mathrm{kyr}, 1.032 \mathrm{~m} / \mathrm{kyr}$, and $1.452 \mathrm{~m} / \mathrm{kyr}$, respectively.

From this and simply by looking at Eq. 53 we see that the expansion rate increases with larger orbit size, so that the orbit growth is actually accelerating. This likely continues until the component separation is so large that the gravitational interaction of lofted particles with the secondary weakens to the point that the mechanism for angular momentum transfer demonstrated herein must shut down. But once having reached very large separation the likelihood of the secondary being stripped away from the primary by perturbations due to solar gravity interaction and planetary flyby greatly increases. The end state for the system's evolution is then a pair of asteroids with similar heliocentric orbits that may be considered a "divorced" binary pair. Recently, observational evidence for such pairs among NEOs and main-belt asteroids has been obtained from new and archived heliocentric asteroid orbit data (Vokrouhlický and Nesvorný, submitted for publication).

Meanwhile, as the system is separating, one can safely assume the same YORP angular acceleration continues to act on the primary (though perhaps resurfacing and figure regularization due to the lofting of material from it's surface may gradually change the YORP coefficient and reduce YORP-driven angular acceleration). In this case the primary will continue to spin up but with loss of the regulating effect against spin-up at large separation, so a transition similar to the first seen in the very high applied angular acceleration case output shown in the last section may occur. From the close ring of debris then levitated around the equator and particles thrown off to successively higher altitudes, it is conceivable that enough material may eventually be shed to accumulate and form into another distinct body well interior to the orbit of the departing secondary in the (prior) system. If this in fact occurs before the older secondary has been stripped away, a triple system with a very much closer and smaller younger tertiary body exists for a certain time, before that body becomes the new secondary, and the new system repeats a similar overall evolution. The body size ratios and relative orbit sizes obtained from recent radar imaging of the triple NEA system (153591) 2001 SN 263 may suggest such a scenario (Nolan et al., 2008).

Similar conclusions to the above regarding the end state for the system's evolution and the possibility of a new secondary's creation are reached in Harris et al. (in preparation). If the overall evolutionary path for binary asteroids is as described above, new campaigns to find systems at each stage along that path and characterize where they are along it are of considerable interest.

\section{Conclusions and Future Work}

In this work we have employed various numerical tools to simulate particle motion within an already well-characterized binary asteroid system which typifies members of the large general class of asynchronous binaries identified in the solar system. We have first performed full-detail dynamic simulation of the binary components themselves, followed by full-detail dynamic simulation of particles in the binary. We initially positioned these on the surface of the primary near the equator, at locations where our analysis showed lofting motion is most likely to first occur with increasing primary spin rate, while we also used other system parameters found to be best suited for such lofting motion to begin. We processed the results of the full-detail dynamic simulation for final particle outcomes (and whether those were expected or not by energy considerations), plus key data probabilistically describing where particles go in the phase space upon lofting. Then we employed a novel probabilistic propagation algorithm grounded in that data but with low computational cost, which captures the important features of the system response to the accumulated particle motion as well. With this method we have clearly demonstrated the hypothesized transfer of angular momentum, injected into the system through YORP-torque on the primary, from primary spin into the mutual orbit. We have directly shown that this angular momentum transfer not only maintains the primary 
near the critical spin rate but leads to accelerating orbit expansion toward eventual binary separation. Our exploration of various properties of the population of loose surface material available to undergo lofting motion, and various hypothetical values of the strength of the external non-gravitational torque on the primary, also yields interesting results. This provides further validation of our methodology as robust to parameter changes and extensible to other interesting cases, even if those do not naturally occur.

Yet there are many open questions brought up by our study and areas in which to improve the modelling of the system dynamics. To begin with, we recognize that although we thoroughly identified the system parameters and location for which particle lofting motion is most likely, the choice of threshold spin rates following from that, at which such motion is said to "activate", is rather arbitrary. Instantly activating such motion precisely when a fixed threshold is crossed is also itself unrealistic. It is of interest to better characterize the conditions under which motion begins for surface material in the identified lofting regions on the primary, accounting for actual physical particle size distribution and contact and friction between particles. For example, some level of cohesion between material at the surface and material lying below it, closer to the centroid, may allow for no motion by any of the material until well above the critical spin rate (as we've locally computed it for that location, simply by considering the surface-normal component of total acceleration). Then the entire mass will suddenly give way above the critical spin rate, with different particle trajectories resulting. Stochastic modelling of lofting onset, particle-by-particle, may be an important improvement. In addition, it may be significant to consider collisional and gravitational interactions between particles of material once lofted. We also note that inclusion of surface charging and electrostatic forces between particles and between the surface and particles may be a worthwhile next step.

However, the basic picture of asynchronous binary evolution supported by this work is not likely to be altered with these model fidelity improvements. It is hoped that further observations, especially those producing detailed shape models and precise mutual orbit and dynamic modes determination, should be able to fill in points along the evolutionary path for such systems.

\section{Acknowledgements}

E.G.F. would like to acknowledge that this material is based upon work supported under a National Science Foundation Graduate Research Fellowship. D.J.S. acknowledges support from a NASA grant from the Planetary Geology and Geophysics program. Supercomputers used for some portions of this work were provided by funding from the JPL Office of the Chief Information Officer.

\section{References}

Bellerose, J. and Scheeres, D. J. 2007, 'Stability of equilibrium points in the restricted full three-body problem', Acta Astronautica 60, 141-152.

Benner, L. A. M., and 11 colleagues, 2007, 'Radar Images Of Binary Near-earth Asteroid 2006 VV2', In: AAS/Division for Planetary Sciences Meeting Abstracts, vol. 39, p. 432.

Cuk, M. 2007, 'Formation and destruction of small binary asteroids', The Astrophysical Journal 659, L57L60.

Cuk, M. and Burns, J. A. 2005, 'Effects of thermal radiation on the dynamics of binary NEAs', Icarus 176, 418-431.

Fahnestock, E. G. and Scheeres, D. J. 2006, 'Simulation of the full two rigid body problem using polyhedral mutual potential and potential derivatives approach', Celestial Mechanics and Dynamical Astronomy 96, $317-339$.

Fahnestock, E. G. and Scheeres, D. J. 2008, 'Simulation and analysis of the dynamics of binary Near-Earth Asteroid (66391) 1999 KW4', Icarus 194, 410-435, URL doi:10.1016/j.icarus.2007.11.007.

Harris, A. W., Fahnestock, E. G. and Pravec, P., in preparation, 'On the shapes and spins of "rubble pile" asteroids', Available: http://www.astro.cz/ asteroid/paris/material/binshape.pdf.

Lee, T., Leok, M. and McClamroch, N. H. 2007, 'Lie Group Variational Integrators for the Full Body Problem in orbital mechanics', Celestial Mechanics and Dynamical Astronomy 98, 121-144. 
Nolan, M. C., and 12 colleagues, 2008, '(153591) 2001 SN263', IAU Circular No. 8921.

Nolan, M. C., Howell, E. S., Magri, C., Beeney, B., Campbell, D. B., Benner, L. A. M., Ostro, S. J., Giorgini, J. D. and Margot, J.-L. 2002, '2002 BM 26', IAU Circular No. 7824.

Nolan, M. C., Howell, E. S. and Miranda, G. 2004, 'Radar Images of Binary Asteroid 2003 YT1', In: Bulletin of the American Astronomical Society, vol. 36, pp. 1132+.

Ostro, S. J., and 15 colleagues, 2006, 'Radar imaging of binary Near-Earth Asteroid (66391) 1999 KW4', Science 314, 1276-1280.

Pravec, P. and Harris, A. W. 2007, 'Binary asteroid population 1. angular momentum content', Icarus 190, $250-259$.

Pravec, P., and 56 colleagues, 2006, 'Photometric survey of binary Near Earth Asteroids', Icarus 181, 63-93.

Scheeres, D. J., and 15 colleagues, 2006, 'Dynamical configuration of binary Near-Earth Asteroid (66391) 1999 KW4', Science 314, 1280-1283.

Shepard, M. K., and 12 colleagues, 2006, 'Radar and infrared observations of binary near-Earth Asteroid 2002 CE26', Icarus 184, 198-210.

Taylor, P. A., Margot, J. L., Nolan, M. C., Benner, L. A. M., Ostro, S. J., Giorgini, J. D. and Magri, C. 2006, 'Radar Imaging of Binary Near-Earth Asteroid 2004 DC', In: Bulletin of the American Astronomical Society, vol. 38 , pp. $577+$.

Vokrouhlický, D. and Nesvorný, D., submitted for publication, 'Pairs of asteroids probably of common origin', The Astronomical Journal Available: http://www. astro.cz/ asteroid/paris/material/vokr0221.pdf.

Werner, R. A. and Scheeres, D. J. 1997, 'Exterior gravitation of a polyhedron derived and compared with harmonic and mascon gravitation representations of asteroid 4769 Castalia', Celestial Mechanics and Dynamical Astronomy 65, 313-344.

Werner, R. A. and Scheeres, D. J. 2005, 'Mutual potential of homogenous polyhedra', Celestial Mechanics and Dynamical Astronomy 91, 337-349. 
Table 1: Meaning of values for particle state vector elements

\begin{tabular}{|c|c|}
\hline Integer value & Meaning \\
\hline $1-36$ & $\begin{array}{l}\text { Particle is lying on surface of Alpha, in } 10^{\circ} \text {-wide sector of longitude on } \\
\text { Alpha w.r.t. rotating barycentric frame denoted by value. Such sectors } \\
\text { are numbered counterclockwise viewed from north pole of Alpha, } 1 \text { being } \\
\text { centered on line of syzygy on opposite side from Beta. }\end{array}$ \\
\hline $37-72$ & $\begin{array}{l}\text { Particle has previously or just impacted onto Beta, in } 10^{\circ} \text {-wide sector of } \\
\text { longitude on Beta w.r.t. rotating barycentric frame denoted by value. Such } \\
\text { sectors are numbered counterclockwise viewed from north pole of Beta, } 1 \\
\text { being centered on line of syzygy on the same side as Alpha. }\end{array}$ \\
\hline 73 & Particle has begun a trajectory that will carry it to escape from the system. \\
\hline 74 & $\begin{array}{l}\text { Particle has begun a trajectory that will leave it still lingering in "orbit" } \\
\text { after very long time. }\end{array}$ \\
\hline $100-9999$ & $\begin{array}{l}\text { Particle will later impact into a sector of longitude on Alpha or on Beta, as } \\
\text { defined above and given by buffered value, but is currently in flight, with } \\
10000-n \text { time intervals past the current time left until impact occurs ( } \\
\text { being the current value). }\end{array}$ \\
\hline 10000 & $\begin{array}{l}\text { Particle is impacting at this time, at sector of longitude given by buffered } \\
\text { value. }\end{array}$ \\
\hline
\end{tabular}

Table 2: Qualitative trajectory outcomes for RF3BP particle simulation output batches, classified by expected outcome based upon the trajectory's initial Jacobi energy level. The table entries are percentages of the total number of particles in each batch (528) reaching the indicated outcome, or the percentage of that number of particles reaching the indicated outcome with the indicated expectation.

\begin{tabular}{|c|c|c|c|c|c|c|c|c|}
\hline$\left\|{ }_{\alpha} \boldsymbol{\omega}_{\alpha}\right\|_{o}(\mathrm{rad} / \mathrm{s})$ & \multicolumn{6}{|c|}{$6.51444 \times 10^{-4}$} & $6.40444 \times 10^{-4}$ & $6.41444 \times 10^{-4}$ \\
\hline Pole offset, $\Delta$ & \multicolumn{2}{|c|}{$0.0^{\circ}$} & \multicolumn{2}{|c|}{$2.5^{\circ}$} & \multicolumn{2}{|c|}{$5.0^{\circ}$} & \multicolumn{2}{|c|}{$2.5^{\circ}$} \\
\hline Lofting side* & same & opposite & same & opposite & same & opposite & same & opposite \\
\hline Return impact & 96.40 & 91.48 & 95.83 & 92.80 & 95.27 & 91.66 & 100 & 100 \\
\hline Expected & 100 & 100 & 100 & 100 & 100 & 100 & 100 & 100 \\
\hline Uncertain & 0 & 0 & 0 & 0 & 0 & 0 & 0 & 0 \\
\hline Rogue (high $J$ ) & 0 & 0 & 0 & 0 & 0 & 0 & 0 & 0 \\
\hline Escape & 0.57 & 0.19 & 1.33 & 0.38 & 0.57 & 0.19 & 0 & 0 \\
\hline Expected & 0 & 0 & 0 & 0 & 0 & 0 & - & - \\
\hline Uncertain & 0 & 0 & 0 & 0 & 0 & 0 & - & - \\
\hline Rogue (low $J$ ) & 100 & 100 & 100 & 100 & 100 & 100 & - & - \\
\hline Transfer impact & 1.89 & 4.92 & 2.08 & 5.30 & 2.65 & 3.98 & 0 & 0 \\
\hline "Orbiting" & 1.14 & 3.41 & 0.76 & 1.52 & 1.52 & 4.17 & 0 & 0 \\
\hline Expected & 0 & 0 & 0 & 0 & 0 & 0 & - & - \\
\hline Rogue $($ low $J$ ) & 100 & 100 & 100 & 100 & 100 & 100 & - & - \\
\hline Rogue (high $J$ ) & 0 & 0 & 0 & 0 & 0 & 0 & - & - \\
\hline
\end{tabular}


Table 3: Several metrics for level and intensity of lofting activity in the binary system, with variation of probabilistic propagation model parameters, both for the spin-up of the primary and for the amount and discretization of material free to be lofted on the primary's surface.

\begin{tabular}{|c|c|c|c|c|c|c|c|}
\hline $\begin{array}{c}\left\|{ }_{\alpha} \boldsymbol{\alpha}_{\alpha}\right\| \\
\left(\mathrm{rad} / \mathrm{s}^{2}\right)\end{array}$ & $\begin{array}{c}\text { \# of } \\
\text { parti- } \\
\text { cles }\end{array}$ & $\begin{array}{c}\text { total } \\
\text { regolith } \\
\text { mass }(\mathrm{kg})\end{array}$ & $\begin{array}{c}\text { particle } \\
\text { mass } \\
(\mathrm{kg})\end{array}$ & $\begin{array}{c}\text { simulation } \\
\text { duration } \\
\text { (s) }\end{array}$ & $\begin{array}{l}\text { mean time } \\
\text { between } \\
\text { episodes }(\mathrm{s})\end{array}$ & $\begin{array}{c}\text { average } \\
\text { mass } \\
\text { aloft }(\mathrm{kg})\end{array}$ & $\begin{array}{c}\text { average } \\
\text { lofting } \\
\text { rate }(\mathrm{kg} / \mathrm{s})\end{array}$ \\
\hline $1.5 \times 10^{-13}$ & 5000 & $10^{10}$ & $2 \times 10^{6}$ & $2.568 \times 10^{6}$ & 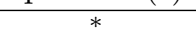 & 494431652.59 & 787708.86 \\
\hline $1.5 \times 10^{-14}$ & 5000 & $10^{10}$ & $2 \times 10^{6}$ & $1.103 \times 10^{7}$ & * & 67278217.80 & 118657.41 \\
\hline $1.5 \times 10^{-15}$ & 5000 & $10^{10}$ & $2 \times 10^{6}$ & $1.015 \times 10^{8}$ & $2.092 \times 10^{5}$ & 528806.13 & 957.84 \\
\hline $1.5 \times 10^{-16}$ & 5000 & $10^{10}$ & $2 \times 10^{6}$ & $9.309 \times 10^{8}$ & $2.912 \times 10^{6}$ & 43697.12 & 78.65 \\
\hline $1.5 \times 10^{-17}$ & 5000 & $10^{10}$ & $2 \times 10^{6}$ & $7.977 \times 10^{9}$ & $2.877 \times 10^{7}$ & 3846.66 & 6.961 \\
\hline $9.5129 \times 10^{-19}$ & 5000 & $10^{10}$ & $2 \times 10^{6}$ & $8.806 \times 10^{10}$ & $5.245 \times 10^{8}$ & 277.98 & 0.5016 \\
\hline $1.5 \times 10^{-19}$ & 5000 & $10^{10}$ & $2 \times 10^{6}$ & $6.440 \times 10^{11}$ & $4.277 \times 10^{9}$ & 35.34 & 0.0634 \\
\hline $1.5 \times 10^{-20}$ & 5000 & $10^{10}$ & $2 \times 10^{6}$ & $3.154 \times 10^{12 * *}$ & $4.139 \mathrm{e} 10$ & 3.113 & 0.00565 \\
\hline $9.5129 \times 10^{-19}$ & 50 & $10^{6}$ & $2 \times 10^{4}$ & $1.873 \times 10^{8}$ & $2.251 \times 10^{5}$ & 177.3 & 0.3256 \\
\hline $9.5129 \times 10^{-19}$ & 500 & $10^{7}$ & $2 \times 10^{4}$ & $3.836 \times 10^{8}$ & $9.290 \times 10^{5}$ & 185.6 & 0.3311 \\
\hline $9.5129 \times 10^{-19}$ & 5000 & $10^{8}$ & $2 \times 10^{4}$ & $2.124 \times 10^{9}$ & $8.696 \times 10^{6}$ & 183.4 & 0.3320 \\
\hline $9.5129 \times 10^{-19}$ & 50000 & $10^{9}$ & $2 \times 10^{4}$ & $1.586 \times 10^{10}$ & $8.424 \times 10^{7}$ & 187.8 & 0.3393 \\
\hline $9.5129 \times 10^{-19}$ & 100 & $10^{8}$ & $1 \times 10^{6}$ & $3.588 \times 10^{9}$ & $1.422 \times 10^{7}$ & 186.4 & 0.3316 \\
\hline $9.5129 \times 10^{-19}$ & 500 & $10^{8}$ & $2 \times 10^{5}$ & $2.317 \times 10^{9}$ & $9.089 \times 10^{6}$ & 184.0 & 0.3363 \\
\hline $9.5129 \times 10^{-19}$ & 1000 & $10^{8}$ & $1 \times 10^{5}$ & $2.130 \times 10^{9}$ & $8.435 \times 10^{6}$ & 183.1 & 0.3328 \\
\hline $9.5129 \times 10^{-19}$ & 5000 & $10^{8}$ & $2 \times 10^{4}$ & $2.124 \times 10^{9}$ & $8.696 \times 10^{6}$ & 183.4 & 0.3320 \\
\hline $9.5129 \times 10^{-19}$ & 10000 & $10^{8}$ & $1 \times 10^{4}$ & $1.982 \times 10^{9}$ & $8.169 \times 10^{6}$ & 182.3 & 0.3304 \\
\hline $9.5129 \times 10^{-19}$ & 50000 & $10^{8}$ & $2 \times 10^{3}$ & $1.970 \times 10^{9}$ & $8.253 \times 10^{6}$ & 183.4 & 0.3322 \\
\hline $9.5129 \times 10^{-19}$ & 5000 & $10^{5}$ & 20 & $1.108 \times 10^{8}$ & $1.083 \times 10^{4}$ & 182.7 & 0.3297 \\
\hline $9.5129 \times 10^{-19}$ & 5000 & $10^{6}$ & 200 & $1.365 \times 10^{8}$ & $8.523 \times 10^{4}$ & 181.8 & 0.3289 \\
\hline $9.5129 \times 10^{-19}$ & 5000 & $10^{7}$ & 2000 & $3.407 \times 10^{8}$ & $8.133 \times 10^{5}$ & 181.8 & 0.3300 \\
\hline $9.5129 \times 10^{-19}$ & 5000 & $10^{8}$ & $2 \times 10^{4}$ & $2.124 \times 10^{9}$ & $8.696 \times 10^{6}$ & 183.4 & 0.3320 \\
\hline $9.5129 \times 10^{-19}$ & 5000 & $10^{9}$ & $2 \times 10^{5}$ & $1.510 \times 10^{10}$ & $7.828 \times 10^{7}$ & 185.7 & 0.3349 \\
\hline $9.5129 \times 10^{-19}$ & 5000 & $10^{10}$ & $2 \times 10^{6}$ & $8.806 \times 10^{10}$ & $5.245 \times 10^{8}$ & 278.0 & 0.5016 \\
\hline
\end{tabular}

* No separation time is given because material transitions to lofting more or less continually in these cases.

** This duration is equivalent to $10^{5}$ years, an arbitrary duration limit reached rather than reaching the 10000 time step limit as in all other cases tabulated. 


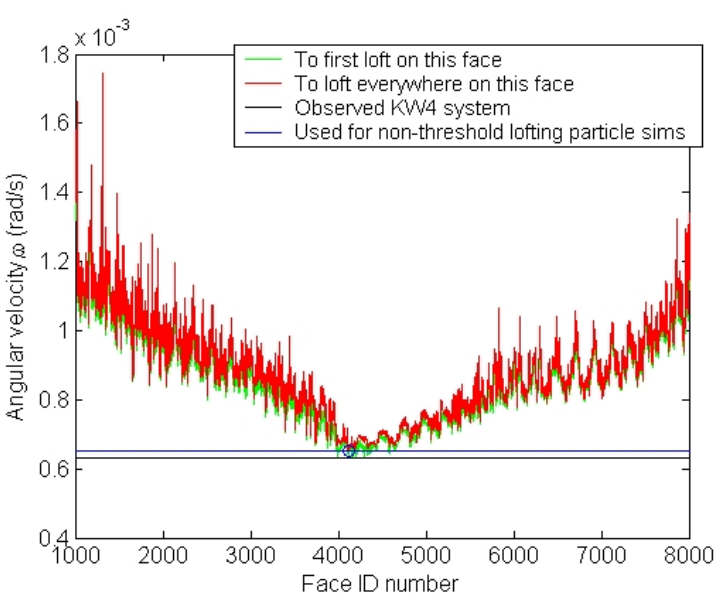

(a)

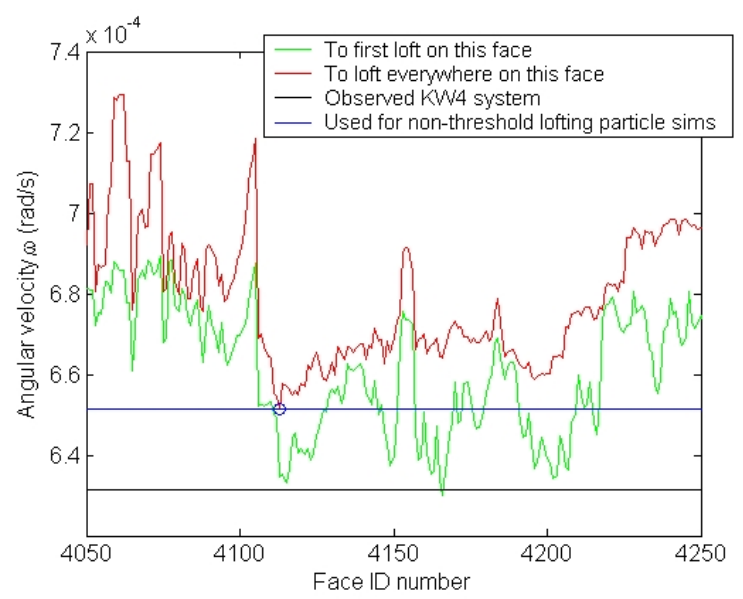

(b)

Figure 1: Spin rates computed for lofting on each facet vs. facet ID \#, computed for uniformly spinning Alpha body mesh by itself, balancing centripetal acceleration with gravitational acceleration. 


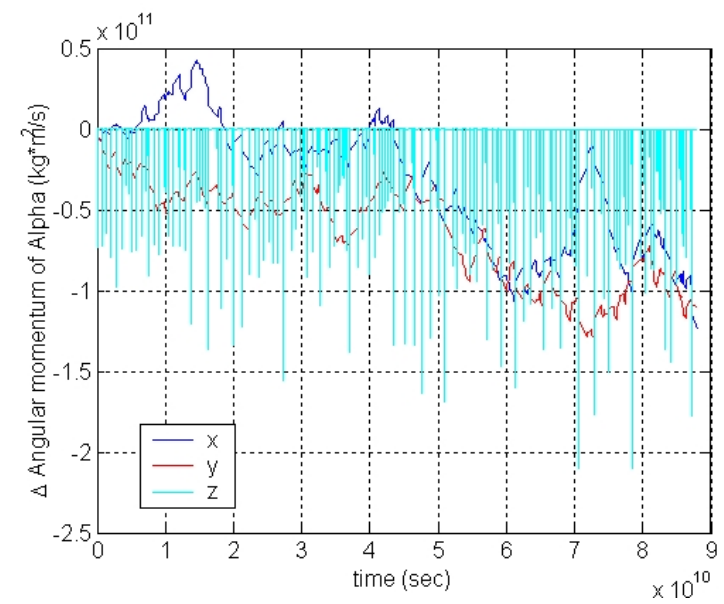

(a) Alpha angular momentum change

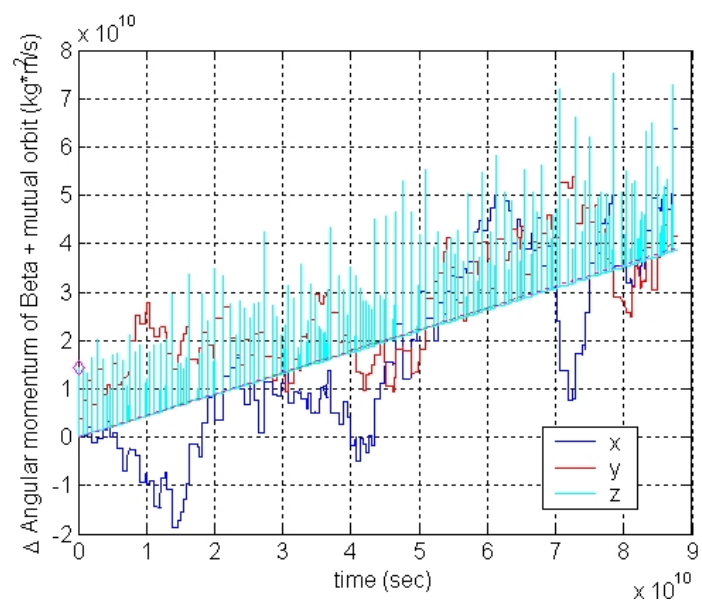

(c) Combined orbit and Beta angular momentum change

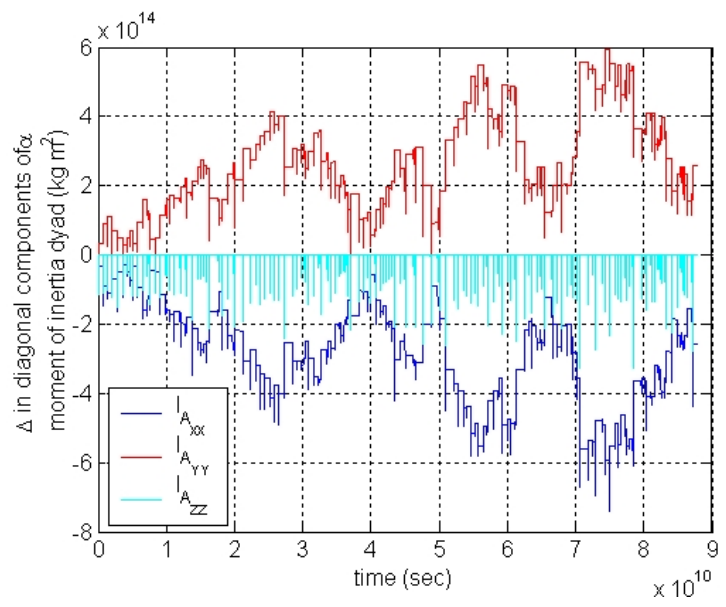

(e) Change in Alpha inertia dyad elements

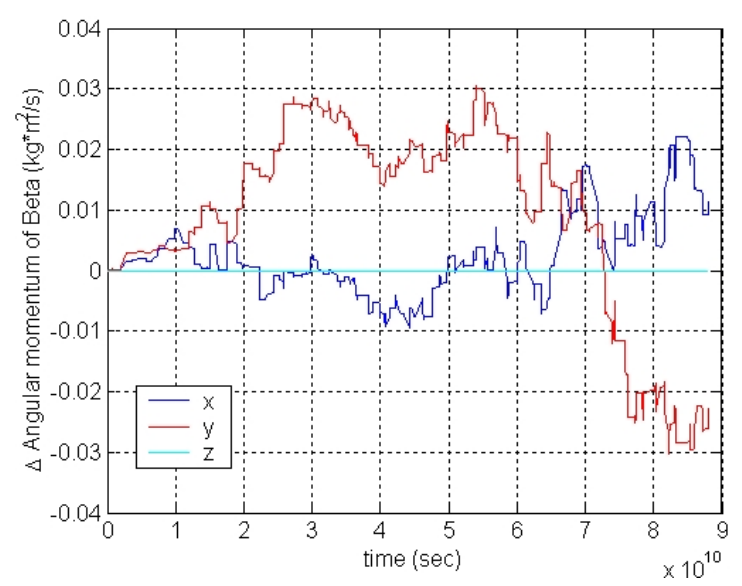

(b) Beta angular momentum change

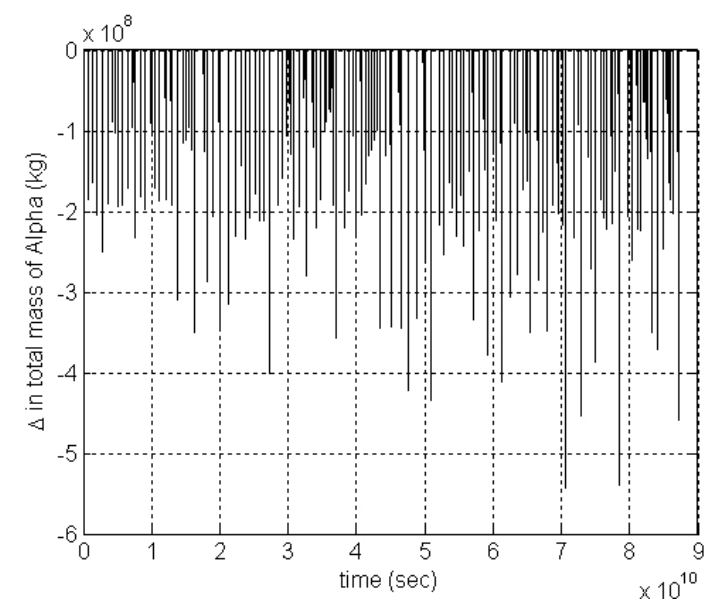

(d) Alpha mass relative to initial value

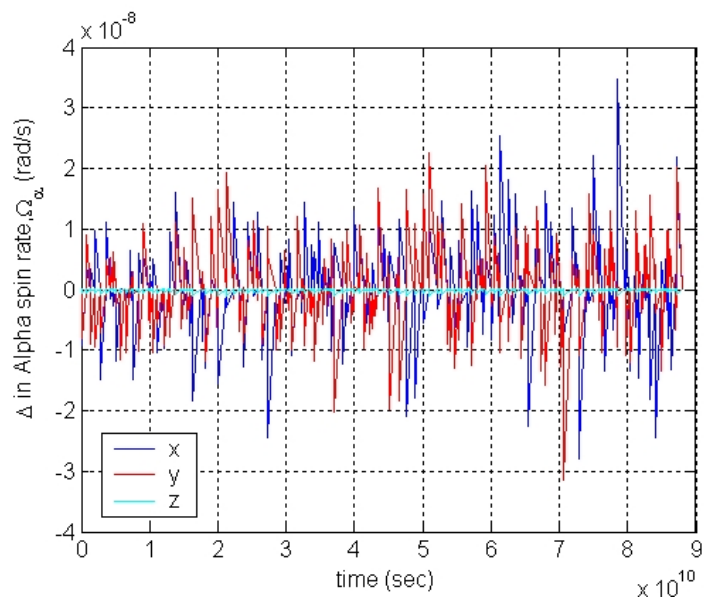

(f) Change in Alpha angular velocity vector

Figure 2: Output of probability based simulation of particles in nominal case with total regolith mass $=1.0 \times 10^{10} \mathrm{~kg}, 5000$ equal-mass particles, applied acceleration $=9.5129 \times 10^{-19} \mathrm{rad} / \mathrm{s}^{2}$. 


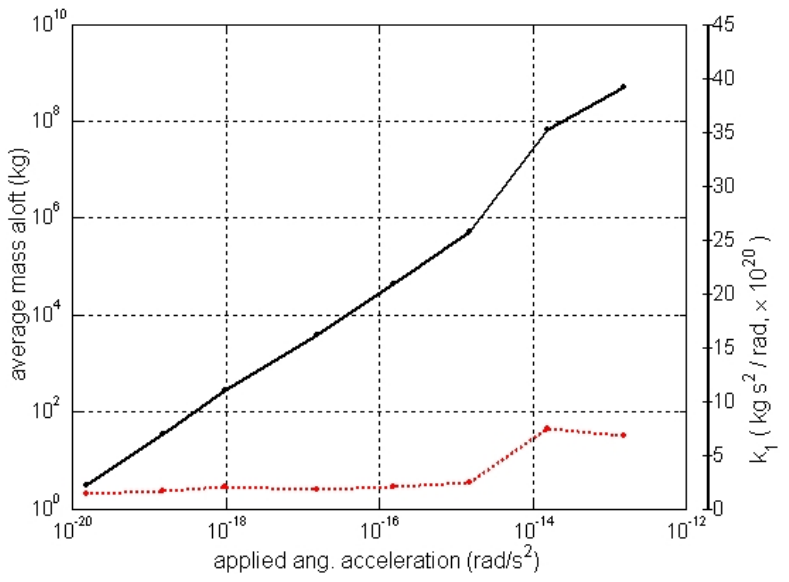

(a) Average mass aloft metric

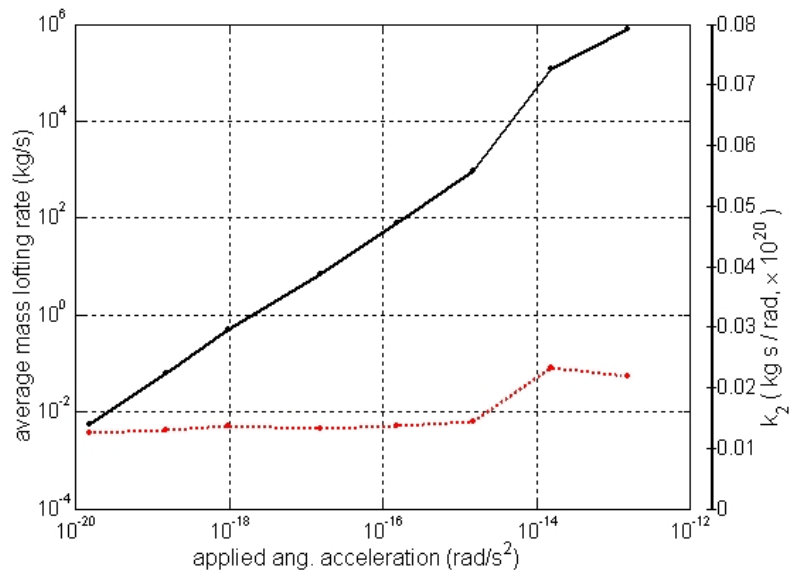

(b) Average mass lofting rate

Figure 3: Trends in the lofting activity metrics versus magnitude of external angular acceleration applied to system primary. The $k_{1}, k_{2}$ quantities are ratios of the metric to the angular acceleration magnitude.

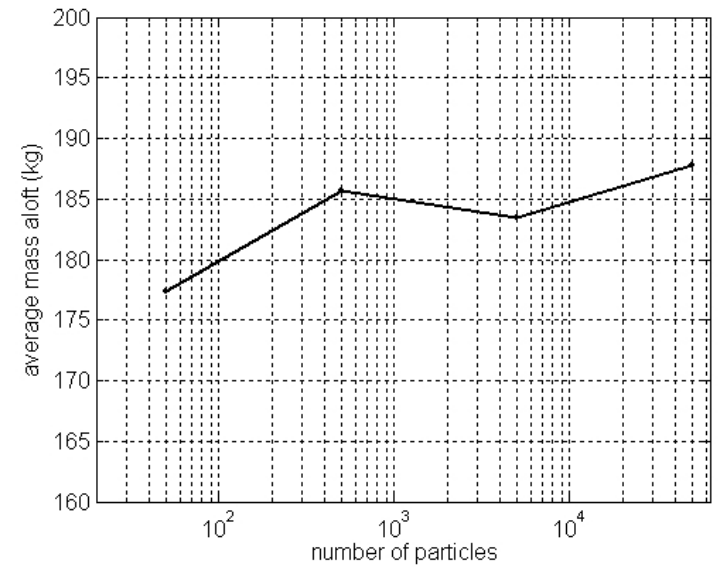

(a) Average mass aloft metric

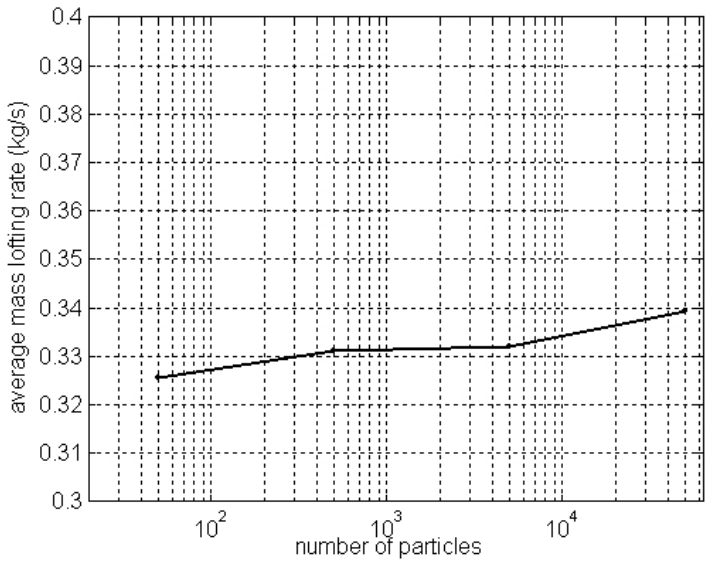

(b) Average mass lofting rate

Figure 4: Trends in the lofting activity metrics versus variation in the number of particles modelled while particle mass (and thus size) is held constant. Total regolith mass varies proportionally with the particle count. The angular acceleration is held constant at the $9.5129 \times 10^{-19} \mathrm{rad} / \mathrm{s}^{2}$ actual YORP level. 


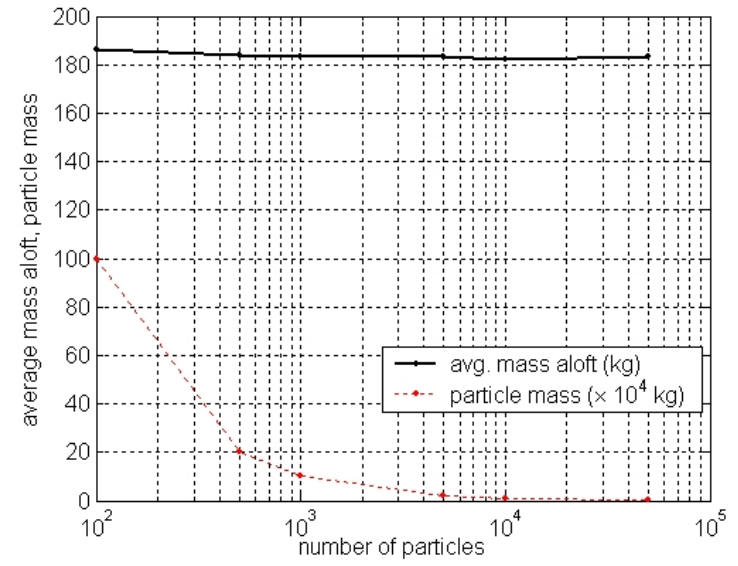

(a) Average mass aloft metric

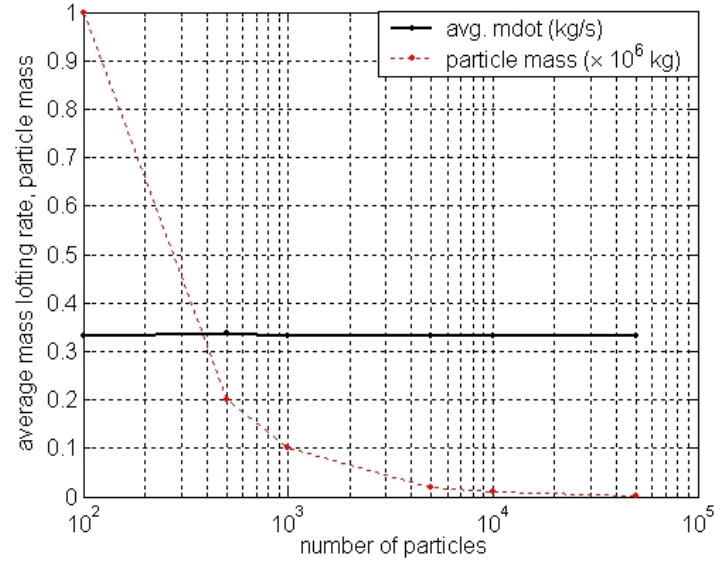

(b) Average mass lofting rate

Figure 5: Trends in the lofting activity metrics with inverse variation in the number of particles modelled and the particle mass (size) while holding total regolith mass constant. The angular acceleration is held constant at the $9.5129 \times 10^{-19} \mathrm{rad} / \mathrm{s}^{2}$ actual YORP level.

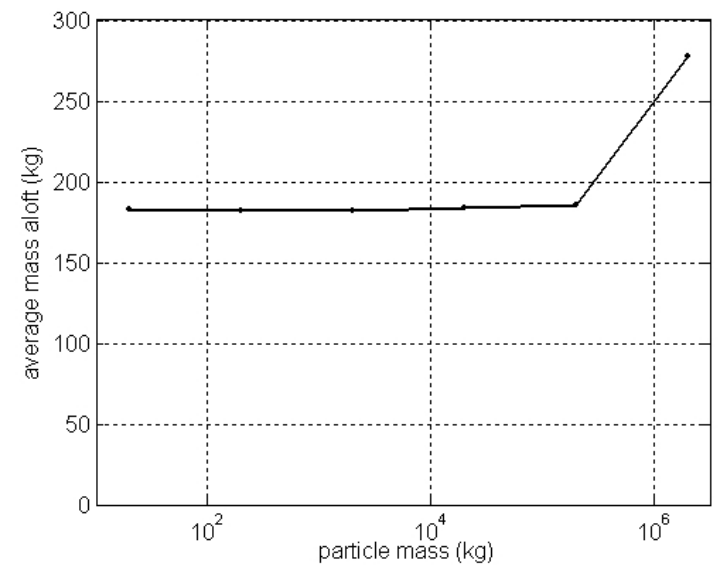

(a) Average mass aloft metric

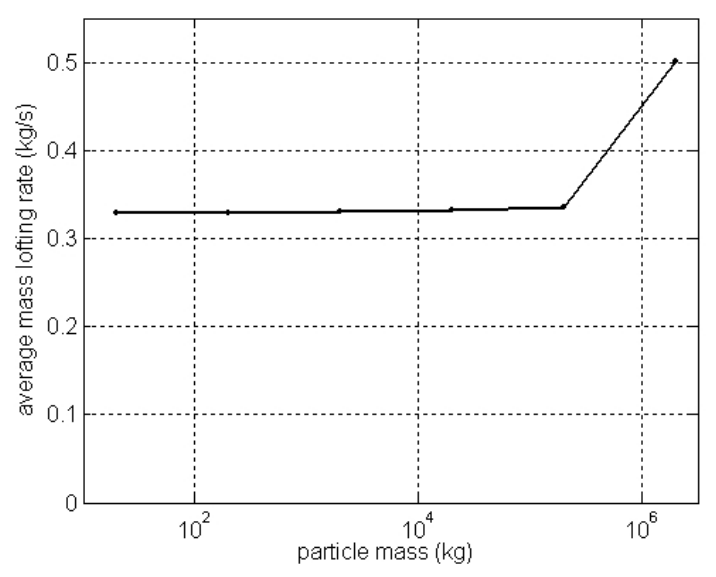

(b) Average mass lofting rate

Figure 6: Trends in the lofting activity metrics versus variation in the particle mass (size) while holding total number of particles constant. Total regolith mass varies proportionally with the particle size. The angular acceleration is held constant at the $9.5129 \times 10^{-19} \mathrm{rad} / \mathrm{s}^{2}$ actual YORP level. 


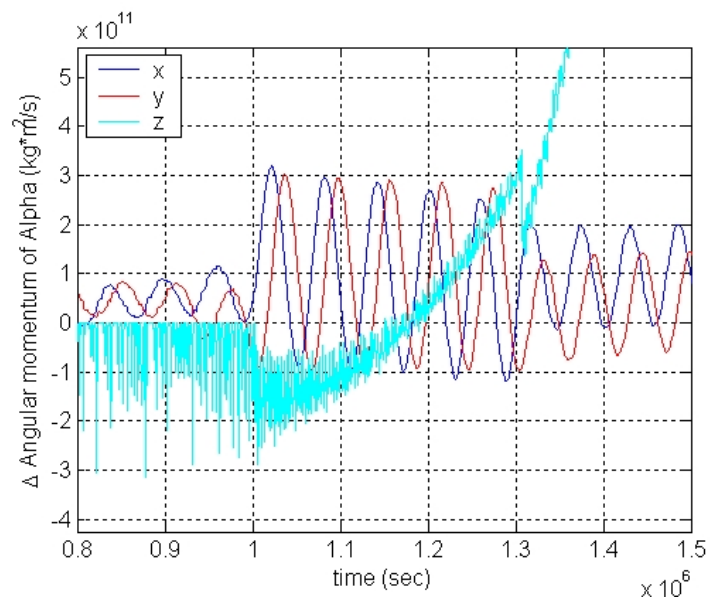

(a) Alpha angular momentum change

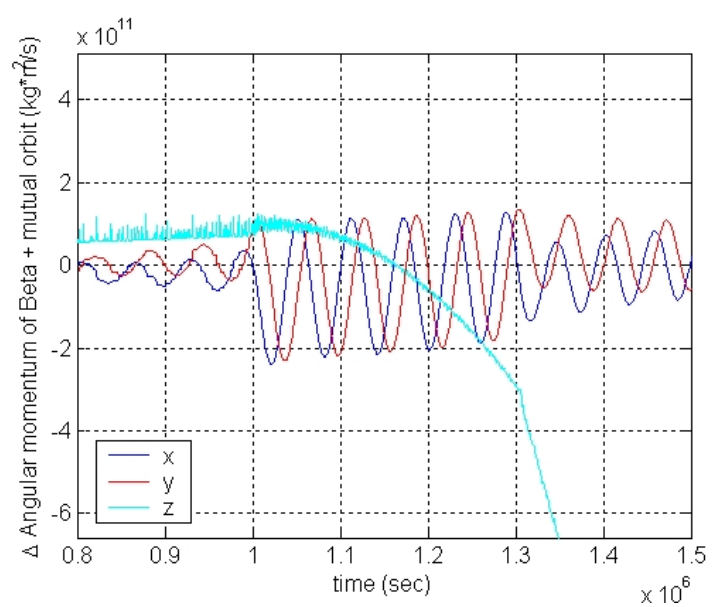

(c) Combined orbit and Beta angular momentum change

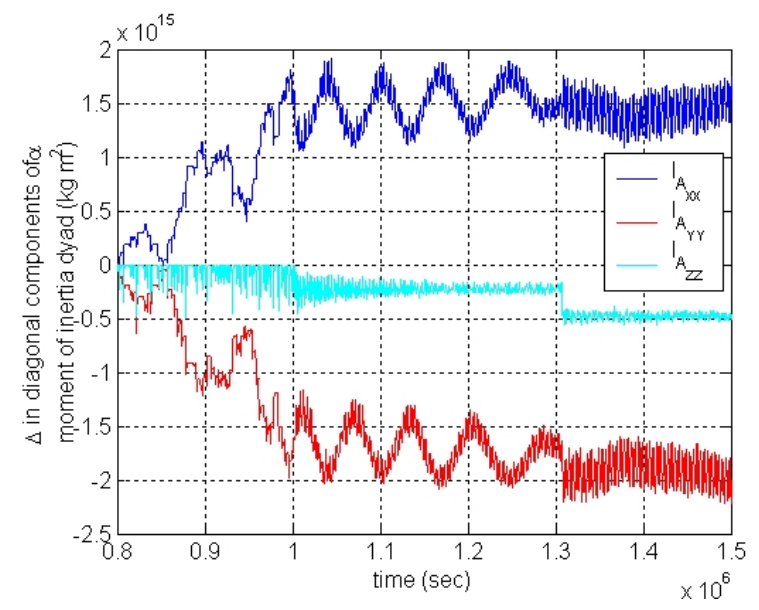

(e) Change in Alpha inertia dyad elements

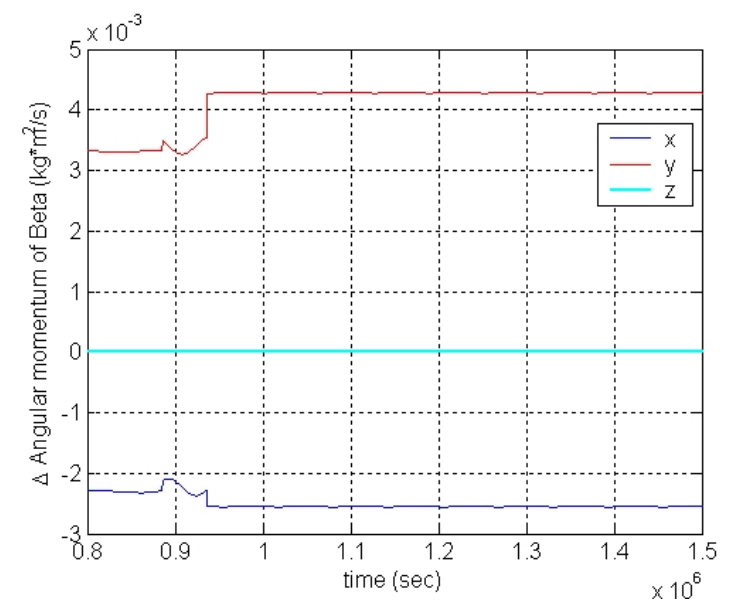

(b) Beta angular momentum change

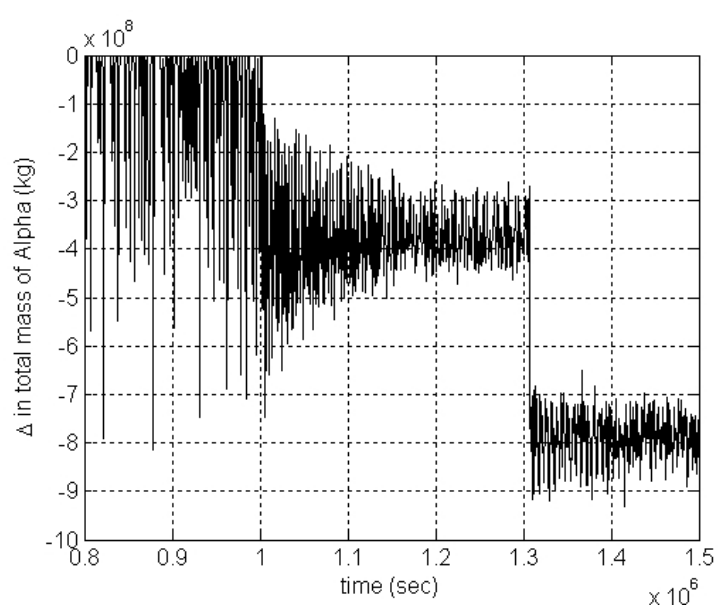

(d) Alpha mass relative to initial value

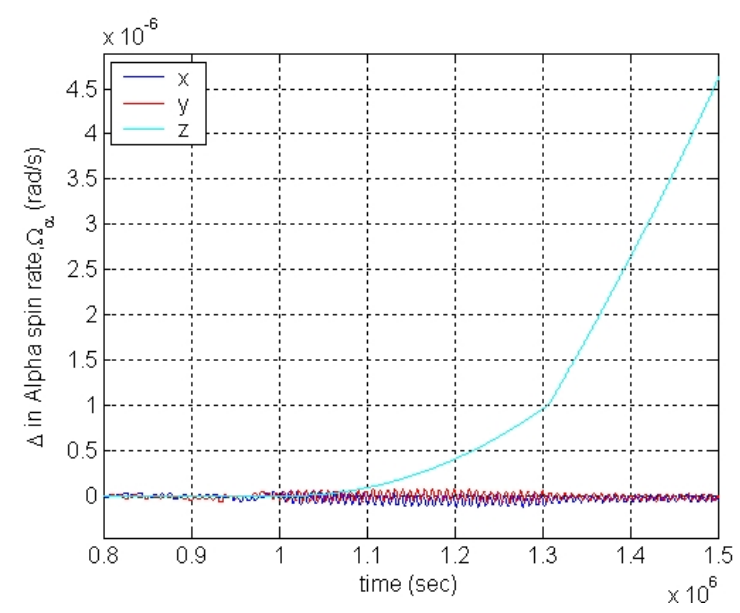

(f) Change in Alpha angular velocity vector

Figure 7: Output of probability based simulation of particles for large acceleration $\left(1.5 \times 10^{-13} \mathrm{rad} / \mathrm{s}^{2}\right)$ case, again with total regolith mass $=1.0 \times{ }^{10} \mathrm{~kg}$ and 5000 equal-mass particles. Note the transitions at approximately $1.0 \times 10^{6}$ and $1.31 \times 10^{6}$ seconds. See text for analysis. 